\title{
Locally constant functions
}

\author{
by
}

Joan Hart and Kenneth K u n e n (Madison, Wis.)

\begin{abstract}
Let $X$ be a compact Hausdorff space and $M$ a metric space. $E_{0}(X, M)$ is the set of $f \in C(X, M)$ such that there is a dense set of points $x \in X$ with $f$ constant on some neighborhood of $x$. We describe some general classes of $X$ for which $E_{0}(X, M)$ is all of $C(X, M)$. These include $\beta \mathbb{N} \backslash \mathbb{N}$, any nowhere separable LOTS, and any $X$ such that forcing with the open subsets of $X$ does not add reals. In the case where $M$ is a Banach space, we discuss the properties of $E_{0}(X, M)$ as a normed linear space. We also build three first countable Eberlein compact spaces, $F, G, H$, with various $E_{0}$ properties. For all metric $M, E_{0}(F, M)$ contains only the constant functions, and $E_{0}(G, M)=C(G, M)$. If $M$ is the Hilbert cube or any infinite-dimensional Banach space, then $E_{0}(H, M) \neq C(H, M)$, but $E_{0}(H, M)=C(H, M)$ whenever $M \subseteq \mathbb{R}^{n}$ for some finite $n$.
\end{abstract}

0. Introduction. If $X$ is a compact Hausdorff space and $M$ is a metric space, let $C(X, M)$ be the space of all continuous functions from $X$ into $M$. $C(X, M)$ is a metric space under the metric induced by the sup norm. $C(X)$ denotes $C(X, \mathbb{R})$, which is a (real) Banach algebra. Following $[5,6,7,14$, 15], if $f \in C(X, M)$, let $\Omega_{f}$ be the union of all open $U \subseteq X$ such that $f$ is constant on $U$. Then $E_{0}(X, M)$ is the set of all $f \in C(X, M)$ such that $\Omega_{f}$ is dense in $X$; these functions are called "locally constant on a dense set". $E_{0}(X)$ denotes $E_{0}(X, \mathbb{R})$.

Clearly, $E_{0}(X)$ is a subalgebra of $C(X)$ and contains all the constant functions. As Bernard and Sidney point out [6, 7, 15], if $X$ is compact metric with no isolated points, then $E_{0}(X)$ is a proper dense subspace of $C(X)$. In this paper, we study the two extreme situations: where $E_{0}(X)$ contains only the constant functions, and where $E_{0}(X)=C(X)$.

A standard example of elementary analysis is a monotonic $f \in C([0,1])$ which does all its growing on a Cantor set; then $f$ is a nonconstant function in $E_{0}([0,1])$. More generally, for "many" $X, E_{0}(X)$ separates points

1991 Mathematics Subject Classification: 46E25, 54D30, 54F45, 03E40, $46 \mathrm{C} 05$.

Authors supported by NSF Grants DMS-9100665 and CCR-9503445. They are grateful to the referee for a number of useful comments on the exposition. 
in $X$, and hence (by the Stone-Weierstrass Theorem), is dense in $C(X)$. Specifically,

0.1. Theorem. If $X$ is compact Hausdorff and $E_{0}(X)$ is not dense in $C(X)$, then

(a) $X$ has a family of $2^{\aleph_{0}}$ disjoint nonempty open subsets.

(b) $X$ is not locally connected.

(c) $X$ is not zero-dimensional.

Part (c) of the Theorem is obvious. Parts (a) and (b) are due to M. E. Rudin and W. Rudin [14], and generalize earlier results of Bernard and Sidney that if $X$ is compact and second countable, then $E_{0}(X)$ is dense in $C(X)$.

First countable, however, is not enough. In $\S 2$, we produce a first countable compact space $X$ such that $E_{0}(X)$ contains only the constant functions. A non-first countable example was constructed in [14]. Our example, patterned after [14], requires some additional complexity, yet we simplify the geometry of the construction by building our space inside a Hilbert space. Our space will be compact in the weak topology, and hence a uniform Eberlein compact space (that is, a weakly compact subspace of a Hilbert space). The approach we describe in $\S 2$ may be used to simplify the construction of [14] as well as to demonstrate that the Rudins' example is a uniform Eberlein compact space. We compare the two constructions further in $\S 2$.

In $\S 3$, we consider the other extreme, and construct a uniform Eberlein compact space $Y$ with no isolated points and with $E_{0}(Y, M)=C(Y, M)$ for all metric spaces $M$. Furthermore, we show that this property can depend on $M$. Although it is easy to see that $E_{0}(X, \mathbb{C})=C(X, \mathbb{C})$ iff $E_{0}(X, \mathbb{R})=$ $C(X, \mathbb{R})$, there is a uniform Eberlein compact space $Z$ such that $E_{0}(Z, \mathbb{R})=$ $C(Z, \mathbb{R})$ but $E_{0}(Z, Q) \neq C(Z, Q)$, where $Q$ is the Hilbert cube.

Of course, there are other compact Hausdorff spaces $X$ which satisfy $E_{0}(X, M)=C(X, M)$ for at least some metric spaces $M$. In $\S 4$, we consider several classes of such spaces $X$. For many familiar compact spaces $X, E_{0}(X, M)=C(X, M)$ for all metric spaces $M$. In addition to the trivial cases where the isolated points are dense in $X, E_{0}(X, M)=C(X, M)$ holds when $X$ is $\beta \mathbb{N} \backslash \mathbb{N}$ or a Suslin line. For some classes of spaces, such as compact ordered spaces and compact extremally disconnected spaces, we present simple necessary and sufficient conditions for $E_{0}(X, M)=C(X, M)$. For some spaces, as in the example of $\S 3$, this can vary with $M$. The detailed discussion of this question entails generalizing the " $E_{0}$ " notion to Baire measurable and Borel measurable maps.

In $\S 5$, we specialize the results of $\S 4$ to the case where $M$ is a Banach space, and consider the properties of $E_{0}(X, M)$ as a normed linear space. In particular, following Bernard and Sidney, $E_{0}(X, M)$ always satisfies the 
Banach-Steinhaus Theorem, although it is also first category in itself in most (but perhaps not all) cases in which it is a proper dense subspace of $C(X, M)$. For example, $E_{0}(X, M)$ is a dense first category subspace of $C(X, M)$ whenever $X$ is a non-trivial infinite product.

In $\S 1$, we prove some preliminary results on Cantor sets used in our construction in $\S 2$.

Bella, Hager, Martinez, Woodward, and Zhou, independently of Bernard and Sidney, defined in $[2,3,12]$ the space $E_{0}(X)$ (they called it $d c(X)$ ), and showed (in the spirit of our Theorem 0.1) that $E_{0}(X)$ is dense in $C(X)$ in many cases. We comment further on their work at the end of $\S 4$.

1. Cantor sets. By a closed interval we mean any compact space homeomorphic to $[0,1] \subseteq \mathbb{R}$. By a Cantor set we mean any space homeomorphic to the usual Cantor set in $\mathbb{R}$; equivalently, homeomorphic to $2^{\omega}$, where $2=$ $\{0,1\}$ has the discrete topology. The following lemma was used also in [14].

1.1. LemMa. If $J$ is a closed interval, $f \in C(J)$, and $f$ is not constant, then there is a Cantor set $H \subset J$ such that $f$ is 1-1 on $H$.

In our construction, we need a uniform version of this. If $H$ is a subset of a product $X \times J$, we use $H_{x}$ to denote $\{y \in J:(x, y) \in H\}$.

1.2. Lemma. Suppose $J$ is a closed interval and $X$ is a compact zerodimensional Hausdorff space, and suppose $f \in C(X \times J)$ is such that for every $x \in X, f \uparrow(\{x\} \times J)$ is not constant. Then there is a set $H \subset X \times J$ such that:

(1) $H_{x}$ is a Cantor set for every $x \in X$.

(2) $f$ is 1-1 on $\{x\} \times H_{x}$ for every $x \in X$.

(3) There is a continuous $\varphi: H \rightarrow 2^{\omega}$ such that the map $(x, y) \mapsto$ $(x, \varphi(x, y))$ is a homeomorphism from $H$ onto $X \times 2^{\omega}$.

Remark. Lemma 1.1 is the special case of Lemma 1.2 where $X$ is a singleton. If we deleted (3), then 1.2 would be immediate from 1.1, using the Axiom of Choice, without any assumption on $X$. But (3) says that we can choose the Cantor sets continuously. As stated, Lemma 1.2 requires $X$ to be zero-dimensional. For example, suppose $X=J=[0,1]$, and take $f$ to be constant on the strip $\{(x, y):|x-y|<1 / 3\}$. Then $H$ must be disjoint from the strip, which is easily seen to contradict (3). Of course, (1) follows from (3).

Lemma 1.1 may be proved by a binary tree argument, and we prove Lemma 1.2 by showing how to build this tree "uniformly" for all $x \in X$. A simpler proof of Lemma 1.1 in [14] takes advantage of the ordering on $\mathbb{R}$, but this proof does not easily generalize to a proof of Lemma 1.2. Moreover, 
the tree argument extends to non-ordered spaces. For example, in Lemma $1.2, J$ could be any compact metric space which is connected and locally connected, and $f$ could be any map into a Hausdorff space.

The following general tree notation will be used here and in $\S \S 2-4$. If $\Delta$ is some index set, then $\Delta^{<\omega}$ denotes the tree of all finite sequences from $\Delta$; this is the complete $\Delta$-ary tree of height $\omega$. For $s \in \Delta^{<\omega}$, let $\operatorname{lh}(s) \in \omega$ be its length. We use () to denote the empty sequence. If $i \leq \operatorname{lh}(s)$, let $s\lceil i$ be the sequence of length $i$ consisting of the first $i$ elements of $s ; t \subseteq s$ iff $t=s\lceil i$ for some $i \leq \operatorname{lh}(s)$. Let $t \alpha$ denote the sequence of length $\operatorname{lh}(t)+1$ obtained by appending $\alpha$ to $t$. Note that $\Delta^{<\omega}$, ordered by $\subseteq$, is a tree with root (), and the nodes immediately above $s$ are the $s \alpha$ for $\alpha \in \Delta$. We say $s, t \in \Delta^{<\omega}$ are compatible iff $s \subseteq t$ or $t \subseteq s$. We let $s \perp t$ abbreviate the statement that $s, t$ are incompatible (not compatible).

A path in $\Delta^{<\omega}$ is a chain, $P$, such that $s \alpha \in P$ implies $s \in P$ for all $s$ and $\alpha$. A path may be empty or finite or countably infinite. The infinite paths are all of the form $\{\psi\lceil n: n \in \omega\}$, where $\psi: \omega \rightarrow \Delta$. In particular, for binary trees, $\Delta=2=\{0,1\}$, and the infinite paths through the Cantor tree, $2^{<\omega}$, are associated with the points in the Cantor set, $2^{\omega}$.

To prove 1.2, fix a metric on $J$. For $E \subseteq J$, let $\operatorname{diam}(E)$ be the diameter of $E$ with respect to this metric. We call a subset of $X \times J$ simple iff it is of the form $\bigcup_{i<k} Q_{i} \times I_{i}$, where $k$ is finite, the $Q_{i}$ for $i<k$ form a disjoint family of clopen sets whose union is $X$, and each $I_{i}$ is a closed interval. We prove 1.2 by iterating the following splitting lemma.

1.3. Lemma. Let $J, X, f$ be as in 1.2 and let $\varepsilon>0$. Then there are simple $A_{0}, A_{1} \subset X \times J$ such that the following hold:

(a) $A_{0} \cap A_{1}=\emptyset$.

(b) For each $x \in X, f\left(\{x\} \times\left(A_{0}\right)_{x}\right) \cap f\left(\{x\} \times\left(A_{1}\right)_{x}\right)=\emptyset$.

(c) For each $x \in X$ and $\mu=0,1, \operatorname{diam}\left(\left(A_{\mu}\right)_{x}\right) \leq \varepsilon$.

(d) For each $x \in X$ and $\mu=0,1, f \uparrow\left(\{x\} \times\left(A_{\mu}\right)_{x}\right)$ is not constant.

Proof. For each $z \in X, f \uparrow(\{z\} \times J)$ is a non-constant map from an interval into an interval, so we may choose disjoint closed intervals $I_{0}(z), I_{1}(z) \subset$ $J$ such that $f\left(\{z\} \times I_{0}(z)\right) \cap f\left(\{z\} \times I_{1}(z)\right)=\emptyset$, diam $\left(I_{\mu}(z)\right) \leq \varepsilon$, and $f \uparrow\left(\{z\} \times I_{\mu}(z)\right)$ is not constant $(\mu=0,1)$. By continuity, there is a neighborhood $U_{z}$ of $z$ such that for all $x \in U_{z}, f\left(\{x\} \times I_{0}(z)\right) \cap f\left(\{x\} \times I_{1}(z)\right)=\emptyset$ and $f \uparrow\left(\{x\} \times I_{\mu}(z)\right)$ is not constant. Since $X$ is compact and zero-dimensional, there are a finite $k$, points $z_{i} \in X(i<k)$, and clopen $Q_{i} \subseteq U_{z_{i}}$ such that the $Q_{i}$ form a partition of $X$. Then let $A_{\mu}=\bigcup_{i<k} Q_{i} \times I_{\mu}\left(z_{i}\right)$.

Proof of 1.2. For $s \in 2^{<\omega}$, choose simple $A_{s} \subseteq X \times J$ such that

(a) For each $s \in 2^{<\omega}, A_{s 0} \cap A_{s 1}=\emptyset$.

(b) For each $x \in X$ and $s \in 2^{<\omega}, f\left(\{x\} \times\left(A_{s 0}\right)_{x}\right) \cap f\left(\{x\} \times\left(A_{s 1}\right)_{x}\right)=\emptyset$. 
(c) For each $x \in X$ and $t \in 2^{<\omega}$, $\operatorname{diam}\left(\left(A_{t}\right)_{x}\right) \leq 1 / \ln (t)$.

(d) For each $x \in X$ and $t \in 2^{<\omega}, f \uparrow\left(\{x\} \times\left(A_{t}\right)_{x}\right)$ is not constant.

We may take $A_{()}=X \times J$; then, for $t=($ ), (c) is vacuous and (d) follows from the hypothesis of 1.2 . Given $A_{s}$, we obtain $A_{s 0}$ and $A_{s 1}$ by applying 1.3 to each box making up $A_{s}$. Let $H=\bigcap_{n \in \omega} \bigcup\left\{A_{s}: \operatorname{lh}(s)=n\right\}$. Let $\varphi(x, y)$ be the (unique) $\psi \in 2^{\omega}$ such that $(x, y) \in A_{\psi \uparrow n}$ for all $n \in \omega$.

2. Making $E_{0}(X)$ small. We describe how to construct a first countable compact space $L_{\omega}$ such that $E_{0}\left(L_{\omega}\right)$ contains only the constant functions. Let $D \subseteq \mathbb{C}$ be the closed unit disk; $D$ will be a subspace of $L_{\omega}$. We shall first focus on the easier task of constructing a space $L_{2}$ such that $D \subset L_{2}$ and each $f \in E_{0}\left(L_{2}\right)$ is constant on $D$. After explaining this, we shall iterate the procedure to produce $L_{\omega}$.

Before we build $L_{2}$, we shall show that every non-constant function $f \in$ $C(D)$ is 1-1 on "many" disjoint Cantor sets. Then, by gluing new disks on those Cantor sets to form $L_{2}$, we can make sure that no such $f$ can extend to a function in $E_{0}\left(L_{2}\right)$.

For $\theta \in[0,2 \pi)$, let $R_{\theta}$ denote the ray $\{z \in D: z \neq 0 \& \arg (z)=\theta\}$. Let $\mathfrak{c}=2^{\aleph_{0}}$.

2.1. LEMmA. If $f \in C(D)$ is non-constant, then there are $\mathfrak{c}$ distinct $\theta$ such that $f$ is non-constant on $R_{\theta}$.

Proof. The set of all such $\theta$ is open.

We identify $\mathfrak{c}$ with a von Neumann ordinal, so that we may use $\mathfrak{c}$ also as an index set.

2.2. Lemma. There is a disjoint family $\left\{K_{\alpha} \subset D \backslash\{0\}: \alpha \in \mathfrak{c}\right\}$ of $\mathfrak{c}$ Cantor sets with the following property: For each non-constant $f \in C(D)$, there is a Cantor set $H_{f} \subset D \backslash\{0\}$ such that $f$ is $1-1$ on $H_{f}$ and such that $A=\left\{\alpha \in \mathfrak{c}: K_{\alpha} \subseteq H_{f}\right\}$ has size $\mathfrak{c}$.

Pr o of. First, applying Lemma 2.1 and transfinite induction, assign to each non-constant $f \in C(D)$ a $\theta_{f} \in[0,2 \pi)$ so that $f$ is non-constant on $R_{\theta_{f}}$ and $\theta_{f} \neq \theta_{g}$ whenever $f \neq g$. Then, applying Lemma 1.1, choose a Cantor set $H_{f} \subset R_{\theta_{f}}$ such that $f$ is $1-1$ on $H_{f}$. Partition each $H_{f}$ into $\mathfrak{c}$ disjoint Cantor sets. Since the $H_{f}$ are all disjoint, this gives us the desired family of $\mathfrak{c} \cdot \mathfrak{c}=\mathfrak{c}$ Cantor sets.

Informally, we now replace each $K_{\alpha}$ by a copy of $K_{\alpha} \times D$, identifying $K_{\alpha} \times\{0\}$ with the old $K_{\alpha}$. For different $\alpha$, we want the $K_{\alpha} \times D$ to point in "perpendicular directions". To make the notion of "perpendicular" formal, we simply embed $L_{2}$ into a Hilbert space. Since we want each "direction" to be a whole disk, we use a complex Hilbert space to simplify the notation. 
One could use a real Hilbert space instead by replacing each unit vector in the following proof by a pair of unit vectors. In either case, the following simple criterion can be used to verify first countability.

2.3. Lemma. If $\mathbb{B}$ is a Hilbert space and $X \subset \mathbb{B}$ is compact in the weak topology, then $X$ is first countable in the weak topology iff, for each $\vec{x} \in X$, there is a countable (or finite) $C_{\vec{x}} \subset \mathbb{B}$ such that no $\vec{v} \in X \backslash\{\vec{x}\}$ satisfies $\vec{x} \cdot \vec{c}=\vec{v} \cdot \vec{c}$ for all $\vec{c} \in C_{\vec{x}}$.

P r o o f. By definition of the weak topology, the stated condition is equivalent to each $\{\vec{x}\}$ being a $G_{\delta}$-set in $X$, which is equivalent to first countability in a compact space.

We remark that the condition of Lemma 2.3 need not imply first countability when $X$ is not weakly compact.

2.4. LEMMA. There is a first countable uniform Eberlein compact space $L_{2}$ such that $D$ is a retract of $L_{2}$ and each $f \in E_{0}\left(L_{2}\right)$ is constant on $D$.

Proof. Let $\mathbb{B}$ be a complex Hilbert space with an orthonormal basis consisting of $\mathfrak{c}$ unit vectors $\vec{e}_{\alpha}$, for $\alpha \in \mathfrak{c}$, together with one more, $\vec{e}$. We identify $D$ with its homeomorphic copy, $D^{\prime}=\{z \vec{e}:|z| \leq 1\} \subset \mathbb{B}$. Let $\pi$ be the perpendicular projection from $\mathbb{B}$ onto the one-dimensional subspace spanned by $\vec{e}$.

Let the $K_{\alpha} \subset D^{\prime} \backslash\{\overrightarrow{0}\}$ be as in Lemma 2.2 (replacing the $D$ there by $D^{\prime}$ ). Let $L_{2}$ be the set of all $\vec{x} \in \mathbb{B}$ that satisfy (1)-(3):

(1) $|\vec{x} \cdot \vec{e}| \leq 1$, and, for each $\alpha \in \mathfrak{c},\left|\vec{x} \cdot \vec{e}_{\alpha}\right| \leq 1 / 2$.

(2) For all distinct $\alpha, \beta$, either $\vec{x} \cdot \vec{e}_{\alpha}=0$ or $\vec{x} \cdot \vec{e}_{\beta}=0$.

(3) For all $\alpha$, either $\vec{x} \cdot \vec{e}_{\alpha}=0$ or $\pi(\vec{x}) \in K_{\alpha}$.

So, points of $L_{2}$ are either of the form $z \vec{e}$, with $|z| \leq 1$, or of the form $z \vec{e}+w \vec{e}_{\alpha}$, where $|z| \leq 1,|w| \leq 1 / 2$, and $z \vec{e} \in K_{\alpha}$. In particular, $D^{\prime}=$ $\pi\left(L_{2}\right) \subset L_{2}$.

We give $L_{2}$ the topology inherited from the weak topology on $\mathbb{B}$. Note that $L_{2}$ is weakly closed. Since $L_{2}$ is also norm bounded, $L_{2}$ is compact. To see that $L_{2}$ is first countable, apply Lemma 2.3 . If $\pi(\vec{x})$ is in no $K_{\alpha}$, set $C_{\vec{x}}=\{\vec{e}\}$, while if $\pi(\vec{x})$ is in some $K_{\alpha}$, this $\alpha$ is unique (by the disjointness of the $K_{\alpha}$ ), and we set $C_{\vec{x}}=\left\{\vec{e}, \vec{e}_{\alpha}\right\}$.

Let $U_{\alpha}=L_{2} \cap \pi^{-1}\left(K_{\alpha}\right) \backslash K_{\alpha}=\left\{\vec{x} \in L_{2}: \vec{x} \cdot \vec{e}_{\alpha} \neq 0\right\}$. Observe:

(i) $U_{\alpha}$ is an open subset of $L_{2}$, but

(ii) For each $\vec{x} \in K_{\alpha}, L_{2} \cap \pi^{-1}(\{\vec{x}\})$ is nowhere dense in $L_{2}$.

Now, suppose $f \in E_{0}\left(L_{2}\right)$. We show that $f$ is constant on $D^{\prime}$. If not, fix a Cantor set $H \subseteq D^{\prime}$ such that $f$ is 1-1 on $H$ and such that $A=\left\{\alpha \in \mathfrak{c}: K_{\alpha} \subseteq\right.$ $H\}$ has size $\mathfrak{c}$. Since $f \in E_{0}\left(L_{2}\right)$, we may, for each $\alpha \in A$, choose a non-empty 
open $W_{\alpha} \subseteq U_{\alpha}$ such that $f$ is constant on $W_{\alpha}$. Then, applying (ii) above, choose two distinct points $\vec{x}_{\alpha}$ and $\vec{y}_{\alpha}$ in $W_{\alpha}$ such that $\pi\left(\vec{x}_{\alpha}\right) \neq \pi\left(\vec{y}_{\alpha}\right)$.

For each $\alpha \in A,\left(\pi\left(\vec{x}_{\alpha}\right), \pi\left(\vec{y}_{\alpha}\right)\right)$ is a point in $\{(\vec{v}, \vec{w}) \in H \times H: \vec{v} \neq \vec{w}\}$, which is a second countable space. Since $A$ is uncountable, these points have a limit point in the same space, so we may fix distinct $\vec{v}, \vec{w} \in H$ and a sequence of distinct elements $\alpha_{n}$ in $A(n \in \omega)$ such that the $\pi\left(\vec{x}_{\alpha_{n}}\right)$ converge to $\vec{v}$ and the $\pi\left(\vec{y}_{\alpha_{n}}\right)$ converge to $\vec{w}$. Hence, in the weak topology of $\mathbb{B}$ and $L_{2}$, the $\vec{x}_{\alpha_{n}}$ converge to $\vec{v}$ and the $\vec{y}_{\alpha_{n}}$ converge to $\vec{w}$. Since $f\left(\vec{x}_{\alpha_{n}}\right)=f\left(\vec{y}_{\alpha_{n}}\right)$, we have $f(\vec{v})=f(\vec{w})$, contradicting that $f$ was $1-1$ on $H$.

A similar use of Cantor sets occurs in the construction in [14], with the following differences: Their $K_{\alpha}$ were not disjoint; in fact, in [14] it appears necessary that every Cantor set gets listed uncountably many times. As a result, the space constructed was not first countable. When one does not care about disjointness, there is no advantage to using a disk, so [14] used an interval where we used $D$. The extra dimension in $D$ lets us prove Lemma 2.2 , which is easily seen to be false of $[0,1]$. Actually, when the $K_{\alpha}$ are disjoint, condition (2) above is redundant, since it follows from (3), but if the $K_{\alpha}$ are not disjoint, (2) is required to guarantee that $L_{2}$ is norm bounded.

Although working within a Hilbert space gives us some geometric insight, it is possible to present all the constructions in this section and the next in a purely topological manner, by working instead inside a product of copies of $D$. We just replace, for example, "the $\vec{e}_{\alpha}$ direction" with "the $\alpha$ th coordinate" in an uncountable product. The weak topology is the same as the usual Tikhonov product topology.

By iterating our construction, we now prove the following theorem.

2.5. TheOREM. There is a first countable uniform Eberlein compact space $L_{\omega}$ such that every function in $E_{0}\left(L_{\omega}\right)$ is constant.

Observe that this is not true for the $L_{2}$ of Lemma 2.4. For example, let $g \in E_{0}(D)$ be non-constant, and define $f$ by $f(\vec{x})=g\left(\vec{x} \cdot \vec{e}_{\alpha}\right)$. Then $f \in E_{0}\left(L_{2}\right)$, and is not constant on $U_{\alpha}$. To prevent such functions from existing, we shall, for each $\alpha$, take disjoint Cantor sets $K_{\alpha \beta} \subset U_{\alpha}$, and, for each $\beta$, attach a new disk going off in a new direction, labeled by a unit vector $\vec{e}_{\alpha \beta}$. This would create a space $L_{3}$. But now, we must iterate this procedure, to take care of functions on these new disks. Iterating $\omega$ times, we have unit vectors $\vec{e}_{t}$ indexed by finite sequences from $\mathfrak{c}$.

To describe $L_{\omega}$, we use the same tree notation as in $\S 1$, where now $\mathfrak{c}$ is our index set. For the rest of this section, let $\mathbb{B}$ be a complex Hilbert space with an orthonormal basis consisting of unit vectors $\left\{\vec{e}_{s}: s \in \mathfrak{c}^{<\omega}\right\}$. We shall use $\vec{e}$ to abbreviate $\vec{e}_{()}$and $\vec{e}_{\alpha}$ to abbreviate $\vec{e}_{(\alpha)}$. Let $\pi_{n}$ be the 
perpendicular projection from $\mathbb{B}$ onto the subspace spanned by $\left\{\vec{e}_{s}: \operatorname{lh}(s)<\right.$ $n\}$. In particular, $\pi_{0}(\vec{x})=\overrightarrow{0}$ for all $\vec{x}$, and $\pi_{1}$ is the projection onto the one-dimensional subspace spanned by $\vec{e}$.

If $\operatorname{lh}(s)=n$, let $D_{s}$ be the set of vectors of the form $\sum_{i \leq n} z_{i} \vec{e}_{s \uparrow i}$, where each $\left|z_{i}\right| \leq 2^{-i}$. Since $D_{s}$ is finite-dimensional, the weak and norm topologies agree on $D_{s}$, and $D_{s}$ is homeomorphic to $D^{n+1}$. In particular, $D_{()}=\{z \vec{e}$ : $|z| \leq 1\}$ plays the role of the $D^{\prime}$ in the proof of Lemma 2.4. Note that if $i \leq n$, then $\pi_{i+1}\left(D_{s}\right)=D_{s \uparrow i}$.

We begin by enumerating enough of the conditions required for the Cantor sets $K_{t}\left(t \in \mathfrak{c}^{<\omega}\right)$ to define $L_{\omega}$. Then, after defining $L_{\omega}$, we prove a sequence of lemmas, adding conditions on the $K_{t}$ as necessary, to show $L_{\omega}$ has the desired properties.

\subsection{BASIC REQUIREMENTS ON THE $K_{t}$.}

(Ra) $K_{()}=\{\overrightarrow{0}\}$.

(Rb) For each $s$, the $K_{s \alpha}$ for $\alpha \in \mathfrak{c}$ are disjoint closed subsets of $D_{s}$, and $\vec{x} \cdot \vec{e}_{s} \neq 0$ for all $\vec{x} \in K_{s \alpha}$.

(Rc) For each $s$ and each $\beta$, if $n=\operatorname{lh}(s)$, then $\pi_{n}\left(K_{s \beta}\right) \subseteq K_{s}$.

In particular, for $s=()$, we have $K_{\alpha} \subset D_{()}$, as in the proof of Lemma 2.4. Now, we iterate that construction by using the $K_{\alpha \beta}, K_{\alpha \beta \gamma}$, etc. The $K_{()}=\{\overrightarrow{0}\}$ plays no role in the definition of $L_{\omega}$, but is included to make some of the notation more uniform. Item (Rc) for $n=0$ says nothing; for $n=1, \pi_{1}\left(K_{\alpha \beta}\right) \subseteq K_{\alpha}$ corresponds to the informal idea above that the $K_{\alpha \beta}$ are chosen inside $U_{\alpha}$.

We shall need to add conditions ( $\mathrm{Rd}),(\mathrm{Re})$ to $(\mathrm{Ra})-(\mathrm{Rc})$ later.

2.7. Definition. $L_{\omega}$ is the set of all $\vec{x} \in \mathbb{B}$ that satisfy (1)-(3):

(1) For each $s,\left|\vec{x} \cdot \vec{e}_{s}\right| \leq 2^{-\operatorname{lh}(s)}$.

(2) For all $s, t$ such that $s \perp t, \vec{x} \cdot \vec{e}_{s}=0$ or $\vec{x} \cdot \vec{e}_{t}=0$.

(3) For all $t$, if $n=\operatorname{lh}(t)$, then either $\vec{x} \cdot \vec{e}_{t}=0$ or $\pi_{n}(\vec{x}) \in K_{t}$.

We give $L_{\omega}$ the weak topology. Set $L_{n}=\pi_{n}\left(L_{\omega}\right)$. For $\vec{x} \in L_{\omega}, P(\vec{x})=\{s \in$ $\left.\mathfrak{c}^{<\omega}: \vec{x} \cdot \vec{e}_{s} \neq 0\right\}$. For $t \in \mathfrak{c}^{<\omega}$ and $n=\operatorname{lh}(t)$, set $U_{t}=L_{\omega} \cap\left(\pi_{n}^{-1}\left(K_{t}\right) \backslash K_{t}\right)$.

2.8. Lemma. Each $L_{n}$ is a closed subset of $L_{\omega}$ and $\bigcup_{n \in \omega} L_{n}$ is dense in $L_{\omega}$.

Pro of. $L_{n} \subseteq L_{\omega}$ holds because each of (1), (2), (3) is preserved under $\pi_{n}$. Density follows because for every $\vec{x} \in \mathbb{B}$, the $\pi_{n}(\vec{x})$ converge weakly (and in norm) to $\vec{x} . L_{n}$ is closed in $L_{\omega}$ because $\pi_{n}(\mathbb{B})$ is weakly closed in $\mathbb{B}$.

We think of the $L_{n}$ as the levels in the construction: $L_{0}=K_{()} ; L_{1}=D_{()}$; $L_{2}$ is exactly the space constructed in the proof of Lemma 2.4. The $U_{t}$ will play the same role here as the $U_{\alpha}$ did there. Elements of $L_{3} \backslash L_{2}$ are of the 
form $r_{0} \vec{e}+r_{1} \vec{e}_{\alpha}+r_{2} \vec{e}_{\alpha \beta}$, where $0<\left|r_{i}\right| \leq 2^{-i}$ for each $i, r_{0} \vec{e} \in K_{\alpha}$, and $r_{0} \vec{e}+r_{1} \vec{e}_{\alpha} \in K_{\alpha \beta}$.

2.9. Lemma. (i) For each $\vec{x} \in L_{\omega}, P(\vec{x})$ is a path in $\mathfrak{c}^{<\omega}$.

(ii) For each $\vec{x} \in L_{\omega},\|\vec{x}\|^{2} \leq 4 / 3$.

(iii) $L_{\omega}$ is weakly closed in $\mathbb{B}$.

(iv) $L_{\omega}$ is first countable and compact.

(v) Each $U_{t}$ is open in $L_{\omega}$.

Proof. For (i), use items (2), (3) in the definition of $L_{\omega}$ and the fact that $\vec{x} \cdot \vec{e}_{s} \neq 0$ for all $\vec{x} \in K_{s \alpha}$. Now, (ii) follows by item (1), since we have $\|\vec{x}\|^{2} \leq \sum_{n=0}^{\infty}\left(2^{-n}\right)^{2}=4 / 3$. (iii) is immediate from the definition of $L_{\omega}$, and compactness of $L_{\omega}$ follows by (iii) and (ii). First countability follows from Lemma 2.3; $C_{\vec{x}}=\left\{\vec{e}_{s}: s \in P(\vec{x})\right\}$, unless $P(\vec{x})$ is finite with maximal element $s$ and $\vec{x} \in K_{s \alpha}$, in which case $C_{\vec{x}}=\left\{\vec{e}_{s}: s \in P(\vec{x})\right\} \cup\left\{\vec{e}_{s \alpha}\right\}$. For (v), note that $U_{t}=\left\{\vec{x} \in L_{\omega}: \vec{x} \cdot \vec{e}_{t} \neq 0\right\}$.

Applying conditions $(\mathrm{Rc})$ and $(\mathrm{Rb})$ on the $K_{s}$, we have the following lemma.

2.10. LemmA. (i) For each $t$, if $n \leq \operatorname{lh}(t)$ and $s=t\left\lceil n\right.$, then $K_{s} \supseteq$ $\pi_{n}\left(K_{t}\right)$.

(ii) Each $K_{t} \subseteq L_{\mathrm{lh}(t)}$.

If the $K_{\alpha}$ are chosen as in the proof of Lemma 2.4, then every $f \in E_{0}\left(L_{2}\right)$ will be constant on $D_{()}$. We must be careful not to destroy this property in choosing the $K_{\alpha \beta}$ and passing to $L_{3}$. In the proof of Lemma 2.4, it was important that each $\pi^{-1}(\{\vec{x}\})$ was nowhere dense. Now, $L_{2} \cap \pi^{-1}(\{\vec{x}\})$ will still be nowhere dense in $L_{2}$, but depending on how the $K_{\alpha \beta}$ meet this set, $L_{3} \cap \pi^{-1}(\{\vec{x}\})$ might have interior points. To handle this, we assume the following product structure on the $K_{s}$ :

(Rd) For each $s$ of length $n \geq 0$ and each $\alpha$, there are a non-empty relatively clopen subset $P \subseteq K_{s}$ and a homeomorphism $\psi$ from $P \times 2^{\omega}$ onto $K_{s \alpha}$, satisfying $\pi_{n}(\psi(\vec{x}, y))=\vec{x}$ for all $\vec{x} \in P$ and all $y \in 2^{\omega}$.

Note that $(\mathrm{Rd})$ implies that $\pi_{n}\left(K_{s \alpha}\right)=P$. Induction on $\operatorname{lh}(s)$ establishes the next lemma.

2.11. Lemma. $K_{s}$ is a Cantor set whenever $\operatorname{lh}(s)>0$.

2.12. Lemma. Suppose that $m>0$ and $C$ is a closed subset of $L_{m}$ such that $C$ is nowhere dense (in the relative topology of $L_{m}$ ) and $C \cap K_{s}$ is nowhere dense (in the relative topology) in $K_{s}$ for all $s$ of length $m$. Then $L_{\omega} \cap \pi_{m}^{-1}(C)$ is nowhere dense in $L_{\omega}$. In particular, $L_{\omega} \cap \pi_{m}^{-1}(\{\vec{x}\})$ is nowhere dense in $L_{\omega}$ for all $\vec{x} \in L_{m}$. 
Pr o of. The "in particular" follows from Lemma 2.11, which implies that $C=\{\vec{x}\}$ satisfies the hypotheses of Lemma 2.12. Now set $C_{n}=L_{n} \cap \pi_{m}^{-1}(C)$ for each $n \geq m$; so $C_{m}=C$. To prove 2.12 , since $\bigcup_{n \in \omega} L_{n}$ is dense in $L_{\omega}$, it suffices to prove claim (i) below. To do this, we prove claims (i) and (ii) together, by induction on $n \geq m$.

(i) For each $n \geq m, C_{n}$ is nowhere dense in $L_{n}$.

(ii) Whenever $\operatorname{lh}(s)=n, C_{n} \cap K_{s}$ is nowhere dense in $K_{s}$.

Claim (ii) for $n+1$ follows from (ii) for $n$ plus assumption (Rd) on the $K_{s}$, and claim (i) for $n+1$ follows from (i) and (ii) for $n$ (just using (Ra)-(Rc)).

For each $s \in \mathfrak{c}^{<\omega}$, with $\ln (s)=n$, let

$$
\widehat{K}_{s}=\left\{\vec{v}+z \vec{e}_{s}: \vec{v} \in K_{s} \&|z| \leq 2^{-n}\right\} .
$$

Note that $\widehat{K}_{s}$ is homeomorphic to $K_{s} \times D$ and is a subset of $L_{\omega}$. If $H \subseteq \widehat{K}_{s}$ and $\vec{v} \in K_{s}$, let $H_{\vec{v}}$ be the "vertical slice", $\left\{\vec{v}+z \vec{e}_{s}:|z| \leq 2^{-n}\right\}$. Call a function $f$ s-level-constant iff $f$ only depends on the $\vec{v}$ here; that is, $f$ is constant on each $\left(\widehat{K}_{s}\right)_{\vec{v}}$. In particular, $f$ is ()-level-constant iff $f$ is constant on $D_{()}$, and the $K_{\alpha}$ chosen as in the proof of Lemma 2.4 will ensure that every $f \in E_{0}\left(L_{\omega}\right)$ is ()-level-constant. Likewise, we shall choose the $K_{s \alpha}$ to ensure that every $f \in E_{0}\left(L_{\omega}\right)$ is $s$-level-constant. Note first that if we do this for all $s$, then $f$ is constant.

2.13. Lemma. If $f \in C\left(L_{\omega}\right)$ is s-level-constant for all $s \in \mathfrak{c}^{<\omega}$, then $f$ is constant.

Proof. By induction on $n, f$ is constant on each $L_{n}$. The result follows because $\bigcup_{n \in \omega} L_{n}$ is dense in $L_{\omega}$.

Now we list the final condition on the $K_{s \alpha}$ :

(Re) For each $s$ of length $n$ and each $f \in C\left(L_{\omega}\right)$ : If $f$ is not $s$-levelconstant, then there are a non-empty clopen set $P \subseteq K_{s}$, a Cantor set $H \subseteq\left\{\vec{v}+z \vec{e}_{s}: \vec{v} \in P \&|z| \leq 2^{-n}\right\}$, and uncountably many different $\alpha$ such that $K_{s \alpha} \subset H$, and for each $\vec{v} \in P, f$ is $1-1$ on $H_{\vec{v}}$.

We must verify that we may choose the $K_{t}$ to meet all five conditions (Ra)-(Re). We choose these by induction on $\operatorname{lh}(t)$. Condition (Ra) specifies $K_{()}$, and the $K_{\alpha}$ will be exactly as in the proof of Lemma 2.4; these were chosen by applying Lemma 2.2. Likewise, given $K_{s}$ with $\operatorname{lh}(s)>0$, we choose the $K_{s \alpha}$ by applying Lemma 2.14 below to $\widehat{K}_{s}$. In fact, we modify the proof of Lemma 2.2, replacing Lemma 1.1 by Lemma 1.2, to prove this lemma.

2.14. Lemma. Let $\left\{E_{\delta}: \delta \in \mathfrak{c}\right\}$ be a partition of $2^{\omega}$ into $\mathfrak{c}$ Cantor sets. If $K$ is a Cantor set, then there is a disjoint family $\left\{K_{\alpha} \subset K \times(D \backslash\{0\})\right.$ : $\alpha \in \mathfrak{c}\}$ of $\mathfrak{c}$ Cantor sets with the following property: For each $f \in C(K \times D)$ 
with $f \uparrow(\{x\} \times D)$ non-constant for some $x \in K$, there are a non-empty clopen $P \subseteq K$ and an $H \subset P \times(D \backslash\{0\})$ that satisfy conditions (1)-(4):

(1) $H_{x}$ is a Cantor set for every $x \in P$.

(2) $f$ is 1-1 on $\{x\} \times H_{x}$ for every $x \in P$.

(3) There is a continuous $\varphi: H \rightarrow 2^{\omega}$ such that the map $(x, y) \mapsto$ $(x, \varphi(x, y))$ is a homeomorphism from $H$ onto $P \times 2^{\omega}$.

(4) For each $\delta \in \mathfrak{c}$, the set $\left\{(x, y) \in H: \varphi(x, y) \in E_{\delta}\right\}$ is one of the $K_{\alpha}$.

Proof. First, for each such $f$, apply continuity to choose a non-empty clopen $P_{f} \subseteq K$ such that for $\mathfrak{c}$ different $\theta \in[0,2 \pi), f \uparrow\left(\{x\} \times R_{\theta}\right)$ fails to be constant for all $x \in P_{f}$. Then, by transfinite induction, choose a distinct $\theta_{f}$ for each such $f$ such that $f \uparrow\left(\{x\} \times R_{\theta_{f}}\right)$ is not constant for all $x \in P_{f}$. Then choose $H_{f} \subseteq P_{f} \times R_{\theta_{f}}$ such that (1)-(3) hold; this is possible by Lemma 1.2. Of course, $\varphi=\varphi_{f}$ depends on $f$. Finally, let the $K_{\alpha}$ enumerate all the sets $\left\{(x, y) \in H_{f}: \varphi_{f}(x, y) \in E_{\delta}\right\}$ as $f$ and $\delta$ vary.

Now we complete the proof of Theorem 2.5.

Proof of Theorem 2.5. Construct $L_{\omega}$ as above. Suppose $f \in$ $E_{0}\left(L_{\omega}\right)$. By Lemma 2.13, it suffices to prove that $f$ is $s$-level-constant for each $s$. Suppose not. Fix $H, P$ as in condition (Re) above, so that $A=\left\{\alpha: K_{s \alpha} \subseteq H\right\}$ is uncountable. For $\alpha \in A$, choose a non-empty open $W_{\alpha}$ such that $W_{\alpha} \subseteq \bar{W}_{\alpha} \subseteq U_{s \alpha}$ and $f$ is constant on $\bar{W}_{\alpha}$. Then $\pi_{n+1}\left(\bar{W}_{\alpha}\right) \subseteq K_{s \alpha} \subseteq H$ and $\pi_{n}\left(\bar{W}_{\alpha}\right) \subseteq \pi_{n}\left(K_{s \alpha}\right) \subseteq P \subseteq K_{s} \subseteq L_{n}$. Choose $\vec{x}_{\alpha}$ and $\vec{y}_{\alpha}$ in $\bar{W}_{\alpha}$ such that $\pi_{n}\left(\vec{x}_{\alpha}\right)=\pi_{n}\left(\vec{y}_{\alpha}\right)$ but $\pi_{n+1}\left(\vec{x}_{\alpha}\right) \neq \pi_{n+1}\left(\vec{y}_{\alpha}\right)$; this is possible because $\pi_{n+1}\left(\bar{W}_{\alpha}\right)$ is closed in $K_{s \alpha}$ and, by Lemma 2.12 , is not nowhere dense in $K_{s \alpha}$. As in the proof of Lemma 2.4, there are distinct $\vec{v}, \vec{w} \in H$ and a sequence of distinct elements $\alpha_{k}$ in $A(k \in \omega)$ such that the $\pi_{n+1}\left(\vec{x}_{\alpha_{k}}\right)$ converge to $\vec{v}$ and the $\pi_{n+1}\left(\vec{y}_{\alpha_{k}}\right)$ converge to $\vec{w}$. Then, in the weak topology, the $\vec{x}_{\alpha_{k}}$ converge to $\vec{v}$ and the $\vec{y}_{\alpha_{k}}$ converge to $\vec{w}$. So, $f(\vec{v})=f(\vec{w})$, while $\pi_{n}(\vec{v})=\pi_{n}(\vec{w}) \in \pi_{n}(H) \subseteq P$, contradicting that $f$ is 1-1 on $H_{\pi_{n}(\vec{v})}$.

Finally, we remark on $E_{0}(X, M)$ for other $M$.

2.15. Lemma. If $X$ is a compact Hausdorff space and $M$ is any Hausdorff space, then

(1) $E_{0}(X, \mathbb{R})$ contains only the constant functions implies

(2) $E_{0}(X, M)$ contains only the constant functions.

If $M$ contains a closed interval, then (2) implies (1).

Proof. For $(1) \rightarrow(2)$, fix $f \in E_{0}(X, M)$. We may assume $M=f(X)$, whence $M$ is compact. For each $g: M \rightarrow[0,1], g \circ f$ is in $E_{0}(X, \mathbb{R})$ and 
hence constant, which implies that $f$ is constant. For $\neg(1) \rightarrow \neg(2)$, if $g$ maps $\mathbb{R}$ homeomorphically into $M$ and $f$ is a non-constant function in $E_{0}(X, \mathbb{R})$, then $g \circ f$ is a non-constant function in $E_{0}(X, M)$.

In particular, in making $E(X)=E_{0}(X, \mathbb{R})$ small, we also make $E_{0}(X, \mathbb{C})$ small. Note that 2.15 can fail if $M$ does not contain an interval, since then, if $X$ is a closed interval, $E_{0}(X, M)=C(X, M)$ contains only the constant functions (since every arc contains a simple arc), while $E_{0}(X, \mathbb{R})$ is dense in $C(X, \mathbb{R})$. We do not study 2.15 for such $M$ in detail here, but it seems to involve the geometric-topological properties of $X$ and $M$.

3. Making $E_{0}(X)$ big. In Theorem 3.1, we modify the method of $\S 2$ to produce an Eberlein compact space $X$ such that $E_{0}(X)$ is all of $C(X)$. Of course, this is trivially true if the isolated points of $X$ are dense in $X$, but the space we obtain has no isolated points. The space of Theorem 3.1 actually satisfies $E_{0}(X, M)=C(X, M)$ for all metric $M$. However, for a general $X$, this property can vary with $M$. In Theorem 3.2, we show how to get an Eberlein compact space $X$ such that $E_{0}(X, \mathbb{R})=C(X, \mathbb{R})$ but $E_{0}(X, Q) \neq C(X, Q)$, where $Q$ is the Hilbert cube. It is easy to see that $E_{0}(X, \mathbb{R})=C(X, \mathbb{R})$ implies $E_{0}\left(X, \mathbb{R}^{n}\right)=C\left(X, \mathbb{R}^{n}\right)$ for each finite $n$.

3.1. TheOREM. There is a first countable uniform Eberlein compact space $X$ such that $X$ has no isolated points and $E_{0}(X, M)=C(X, M)$ for all metric spaces $M$.

P r o o f. Follow exactly the notation in $\S 2$, so that $X$ will be the $L_{\omega}$ there. Choose sets $K_{t}$ for $t \in \mathfrak{c}^{<\omega}$ so that conditions (Ra)-(Rc) of 2.6 hold, so that all the lemmas through Lemma 2.10 still apply. But, replace (Rd), (Re) by

(Rf) Each $K_{s}$ is a singleton, and the $K_{s \alpha}$, for $\alpha \in \mathfrak{c}$, enumerate all the singletons in $\widehat{K}_{s} \backslash K_{s}$.

As before, $U_{t}=X \cap\left(\pi_{n}^{-1}\left(K_{t}\right) \backslash K_{t}\right)$ where $n=\ln (t)$.

Now, fix $f \in C\left(L_{\omega}, M\right)$, where $M$ is metric.

Note. $f$ is constant on $U_{t}$ for all but countably many $t$. If not, we could find an $s$ and an uncountable $A \subseteq \mathfrak{c}$ such that $f$ is not constant on $U_{s \alpha}$ for all $\alpha \in A$. For $\alpha \in A$, let $K_{s \alpha}=\left\{\vec{x}_{\alpha}\right\}$, and choose $\vec{y}_{\alpha} \in U_{s \alpha}$ such that $f\left(\vec{y}_{\alpha}\right) \neq f\left(\vec{x}_{\alpha}\right)$. Since the range of $f$ is compact, and hence second countable, we may, as in the last paragraph of the proof of Lemma 2.4, fix distinct $p, q \in M$ and distinct $\alpha_{n} \in A(n \in \omega)$ such that the $f\left(\vec{x}_{\alpha_{n}}\right)$ converge to $p$ and the $f\left(\vec{y}_{\alpha_{n}}\right)$ converge to $q$. Now, the points $\vec{x}_{\alpha_{n}}$ are in $K_{s}$, which is compact metric, so, by passing to a subsequence, we may assume that the $\vec{x}_{\alpha_{n}}$ converge to some point $\vec{x} \in K_{s}$. Hence, in the weak topology, since $\pi_{n+1}\left(\vec{y}_{\alpha}\right)=\vec{x}_{\alpha}$, the $\vec{y}_{\alpha_{n}}$ converge to $\vec{x}$ also. Applying $f$ to these sequences, $f(\vec{x})=p \neq q=f(\vec{x})$, a contradiction. 
It follows that $\Omega_{f}$ is dense in $X$, since every non-empty open set in $X$ contains uncountably many $U_{t}$ (to see this, apply the above Note and the fact that the co-zero sets of continuous functions form a basis for $X$ ).

We remark that in the above Note, we used the same method to prove $E_{0}(X)$ big as we used in Lemma 2.4 to prove $E_{0}(X)$ small; we have simply reversed the roles of $f$ and $\pi$.

Also, it is possible to make the space of Theorem 3.1 zero-dimensional by restricting the coordinates to lie in a Cantor set. This would not be possible for the spaces of $\S 2$, or the space used for Theorem 3.2(b) below. The zero-dimensional space was actually already described by van Mill [13] in a different way; he showed that this is an example of a first countable homogeneous Eberlein compact space which is not second countable.

Observe that in the proof of Theorem 3.1, the Hilbert space $\mathbb{B}$ can be either complex or real, since unlike in $\S 2$, we no longer need the $\vec{e}_{s}$ direction to be two-dimensional. This holds in the next construction as well, although we shall need that the base level $L_{0}$ be infinite-dimensional.

Also observe that if the $K_{s \alpha}$ were not singletons, the above proof would establish a modified Note: for all but countably many $t, f(\vec{y})=f\left(\pi_{n}(\vec{y})\right)$ for all $\vec{y} \in U_{t}$. This is the key to building a space satisfying (2) but not (3). We shall make sure that $\widehat{K}_{s}$ has "large dimension", so that any real-valued function will be constant on many subsets of $\widehat{K}_{s}$, and these subsets will be the $K_{s \alpha}$; this will ensure that $E_{0}(X, \mathbb{R})=C(X, \mathbb{R})$. However, if $M$ itself has "large dimension", then this argument will fail, so that $E_{0}(X, M) \neq$ $C(X, M)$.

The following definition and theorem pin down precisely for which $M$ we can conclude $E_{0}(X, M)=C(X, M)$ from $E_{0}(X, \mathbb{R})=C(X, \mathbb{R})$. It suffices to consider only compact $M$, since the range of each continuous map is compact. Let $\mathcal{F}_{0}$ be the collection of all zero- or one-point spaces. For an ordinal $\alpha>0$, let $\mathcal{F}_{\alpha}$ be the class of all compact metric spaces $M$ such that there is a $\varphi \in C(M,[0,1])$ with $\varphi^{-1}\{r\} \in \bigcup_{\delta<\alpha} \mathcal{F}_{\delta}$ for every $r \in[0,1]$. So, for example, induction on $n \in \omega$ shows that $[0,1]^{n} \in \mathcal{F}_{n}$. Then, if $M$ is the one-point compactification of the disjoint union of the $[0,1]^{n}$, we may let $\varphi$ map $M$ to a simple sequence to conclude that $M \in \mathcal{F}_{\omega}$. Define $\mathcal{F}=\bigcup_{\delta \in O N} \mathcal{F}_{\delta}$, where $O N$ is the class of ordinals. Actually, since every compact metric space has at most $\mathfrak{c}$ closed subspaces, $\mathcal{F}=\bigcup_{\delta<\mathfrak{c}^{+}} \mathcal{F}_{\delta}$.

3.2. TheOREM. Let $M$ be any compact metric space.

(a) If $M \in \mathcal{F}, X$ is compact Hausdorff, and $E_{0}(X, \mathbb{R})=C(X, \mathbb{R})$, then $E_{0}(X, M)=C(X, M)$.

(b) If $M \notin \mathcal{F}$, then there is a first countable uniform Eberlein compact space $X_{M}$ with $E_{0}\left(X_{M}, \mathbb{R}\right)=C\left(X_{M}, \mathbb{R}\right)$ but $E_{0}\left(X_{M}, M\right) \neq C\left(X_{M}, M\right)$. 
In Corollary 3.12, we strengthen (b) to the existence of a single $X$ which works for all $M \notin \mathcal{F}$. Of course, this theorem is uninteresting unless we can produce a compact metric space $M \notin \mathcal{F}$, but that follows by a theorem of Levshenko. There is a class of strongly infinite-dimensional spaces which includes the Hilbert cube, $[0,1]^{\omega}$. Levshenko showed that if $M$ is a strongly infinite-dimensional compact metric space and $\varphi \in C(M,[0,1])$, then some $\varphi^{-1}\{r\}$ is strongly infinite-dimensional (see [1]). This gives us the following lemma, which is easily proved by induction on the ordinals.

\subsection{Lemma. If $M \in \mathcal{F}$, then $M$ is not strongly infinite-dimensional.}

The definition of $\mathcal{F}$ also gives us the following easy inductive proof of Theorem 3.2(a).

Proof of Theorem 3.2(a). Suppose that $M \in \mathcal{F}_{\alpha}$, and suppose (inductively) that the result holds for all $M^{\prime} \in \bigcup_{\delta<\alpha} \mathcal{F}_{\delta}$. Suppose $X$ is compact Hausdorff and $E_{0}(X, \mathbb{R})=C(X, \mathbb{R})$. Fix $f \in C(X, M)$. To prove $f \in E_{0}(X, M)$, we fix a non-empty open $U \subseteq X$, and we produce a nonempty open $V \subseteq U$ such that $f$ is constant on $V$. Applying the definition of $\mathcal{F}_{\alpha}$, fix $\varphi \in \bar{C}(M,[0,1])$ such that for each $r \in[0,1]$, we have $\varphi^{-1}\{r\} \in$ $\bigcup_{\delta<\alpha} \mathcal{F}_{\delta}$. Then $\varphi \circ f \in C(X, \mathbb{R})=E_{0}(X, \mathbb{R})$, so fix a non-empty open set $W \subseteq U$ such that $\varphi \circ f$ has some constant value $r$ on $\bar{W}$. Now $\varphi^{-1}\{r\} \in \mathcal{F}_{\delta}$, for some $\delta<\alpha$, and $E_{0}(\bar{W}, \mathbb{R})=C(\bar{W}, \mathbb{R})$ (by the Tietze Theorem; see also Lemma 4.1). Applying the induction hypothesis, $f \backslash \bar{W} \in C\left(\bar{W}, \varphi^{-1}\{r\}\right)=$ $E_{0}\left(\bar{W}, \varphi^{-1}\{r\}\right)$, so we may choose a non-empty open subset $V \subseteq W$ such that $f \nmid V$ is constant.

To prove Theorem 3.2(b), we first prove some more lemmas about $\mathcal{F}$. First, another simple induction yields closure under subsets:

3.4. Lemma. If $M \in \mathcal{F}$ and $H$ is a closed subset of $M$, then $H \in \mathcal{F}$.

We also get closure under finite unions:

3.5. Lemma. Suppose that $M$ is compact metric and $M=H \cup K$, where $H, K$ are closed subsets of $M$ and $H, K \in \mathcal{F}$. Then $M \in \mathcal{F}$.

Proof. Since $H$ is a closed $G_{\delta}$, fix $\varphi \in C(M,[0,1])$ such that $\varphi^{-1}\{0\}=$ $H$. Then, $\varphi^{-1}\{0\} \in \mathcal{F}$. For $r>0$, we have $\varphi^{-1}\{r\} \subseteq K$, so $\varphi^{-1}\{r\} \in \mathcal{F}$ by Lemma 3.4. Thus, $\varphi^{-1}\{r\} \in \mathcal{F}$ for each $r \in[0,1]$, which implies that $M \in \mathcal{F}$.

We say that $M$ is nowhere in $\mathcal{F}$ iff $M$ is non-empty and for each nonempty open $V \subseteq M$, we have $\bar{V} \notin \mathcal{F}$. Note that such an $M$ has no isolated points, since $\mathcal{F}$ contains all one-point spaces.

3.6. Lemma. If $M$ is a compact metric space and $M \notin \mathcal{F}$, then there is a closed set $K \subseteq M$ such that $K$ is nowhere in $\mathcal{F}$. 
Proof. Let $\mathcal{U}=\{U \subseteq M: U$ is open and $\bar{U} \in \mathcal{F}\}$, and let $K=M \backslash \bigcup \mathcal{U}$.

First, note that $K$ is non-empty: If $K$ were empty, then, by compactness, $M$ would be covered by a finite subfamily of $\mathcal{U}$, which would imply $M \in \mathcal{F}$ by Lemma 3.5.

To prove that $K$ is nowhere in $\mathcal{F}$, it suffices (by Lemma 3.4) to prove that $\overline{B(p, \varepsilon)} \cap K \notin \mathcal{F}$ whenever $p \in K$ and $\varepsilon>0$. Note that $B(p, \varepsilon)$ and its closure are computed in $M$, not $K$. Let $N=\overline{B(p, \varepsilon)}$. Fix $\varphi \in C(N,[0,1])$ such that $\varphi^{-1}\{0\}=N \cap K$. Since $B(p, \varepsilon) \cap K$ is non-empty, $N \notin \mathcal{F}$, so there must be some $r \in[0,1]$ such that $\varphi^{-1}\{r\} \notin \mathcal{F}$. However, for $r>0, \varphi^{-1}\{r\}$ is compact and disjoint from $K$, so it is covered by a finite subfamily of $\mathcal{U}$, and hence, as above, is in $\mathcal{F}$. So, $r$ must be 0 , so $N \cap K \notin \mathcal{F}$.

Let $\mathcal{N}(K)$ be the family of all compact $H \subseteq K$ such that $H$ is nowhere in $\mathcal{F}$. The following lemma is trivial, given the above results, but we state it to emphasize the abstract properties of our construction.

3.7. Lemma. If $K$ is compact metric and nowhere in $\mathcal{F}$, then

(1) $\mathcal{N}(K)$ is a family of non-empty closed subspaces of $K$.

(2) $K \in \mathcal{N}(K)$.

(3) For each $H \in \mathcal{N}(K)$ and each non-empty relatively open $U \subseteq H$, there is an $L \in \mathcal{N}(K)$ with $L \subseteq U$.

Most of the proof of Theorem 3.2(b) proceeds using just the conclusion to Lemma 3.7, without any reference to $\mathcal{F}$. Note that if $K$ is a singleton, and $\mathcal{N}(K)$ is redefined to be $\{K\}$, we also have the conclusion to Lemma 3.7 , and the proof of 3.2(b) then reproves Theorem 3.1.

We now construct the space $X_{M}$. Applying Lemma 3.6, let $K$ be a closed subset of $M$ which is nowhere in $\mathcal{F}$. Let $\mathbb{B}$ be a real Hilbert space with an orthonormal basis consisting of unit vectors $\left\{\vec{e}_{s}: s \in \mathfrak{c}^{<\omega}\right\} \cup\left\{\vec{b}_{i}: i \in \omega\right\}$. Let $\mathbb{B}_{n}$ be the closed linear span of $\left\{\vec{e}_{s}: \operatorname{lh}(s)<n\right\} \cup\left\{\vec{b}_{i}: i \in \omega\right\}$. Since $\mathbb{B}_{0}$ is infinite-dimensional, we can embed $K$ in the first level of our space. To do so we replace condition ( $\mathrm{Ra}$ ) of 2.6 by the following:

$\left(\mathrm{Ra}^{\prime}\right) \quad K_{()}$is a weakly compact subset of the closed unit ball of $\mathbb{B}_{0}$, and $K_{()}$is homeomorphic to $K$.

Actually, we could also make $K_{()}$norm compact, but this is unnecessary.

Let $\pi_{n}$ be the perpendicular projection from $\mathbb{B}$ onto $\mathbb{B}_{n}$. If $\operatorname{lh}(s)=n$, let $D_{s}$ be the set of vectors of the form $\vec{v}+\sum_{i \leq n} r_{i} \vec{e}_{s \uparrow i}$, where $\vec{v} \in K_{()}$and each $\left|r_{i}\right| \leq 2^{-i}$. In particular, $D_{()}$is homeomorphic to $K \times[-1,1]$. As in $\S 2$, the product with $[-1,1]$ allows us to make the $K_{\alpha}$ disjoint subsets of $D_{()}$. As before, if $i \leq n$, then $\pi_{i+1}\left(D_{s}\right)=D_{s \uparrow i}$.

We will choose the $K_{t}$ for $t \in \mathfrak{c}^{<\omega}$ so that they satisfy condition $\left(\mathrm{Ra}^{\prime}\right)$, along with (Rb) and (Rc) of 2.6. Now, define $X_{M}=L_{\omega}$ to be the set of 
$\vec{x} \in \mathbb{B}$ satisfying conditions (1)-(3) of 2.7 , along with condition

(0) $\pi_{0}(\vec{x}) \in K_{()}$.

As before, for $t \in \mathfrak{c}^{<\omega}$ and $n=\operatorname{lh}(t), U_{t}=L_{\omega} \cap\left(\pi_{n}^{-1}\left(K_{t}\right) \backslash K_{t}\right)$. So $U_{()}=L_{\omega} \backslash K_{()}=\left\{\vec{x} \in L_{\omega}: \vec{x} \cdot \vec{e} \neq 0\right\}$. In this construction, we still have the levels $L_{n}=\pi_{n}\left(L_{\omega}\right)$, with $L_{0}=K_{()}$and $L_{1}=D_{()}$. Now, elements of level $L_{3} \backslash L_{2}$ are of the form $\vec{v}+r_{0} \vec{e}+r_{1} \vec{e}_{\alpha}+r_{2} \vec{e}_{\alpha \beta}$, where $0<\left|r_{i}\right| \leq 2^{-i}$ for each $i, \vec{v} \in K_{()}, \vec{v}+r_{0} \vec{e} \in K_{\alpha}$, and $\vec{v}+r_{0} \vec{e}+r_{1} \vec{e}_{\alpha} \in K_{\alpha \beta}$.

This $L_{\omega}$ still satisfies Lemmas 2.8-2.10, provided we replace the bound in 2.9(ii) by $7 / 3$. The proofs are the same, except for the proof of 2.9(iv), where we join $\left\{\vec{b}_{i}: i \in \omega\right\}$ to each $C_{\vec{x}}$.

Now, we utilize $\mathcal{N}\left(K_{()}\right)$to choose the $K_{s}$. Choose the $K_{s}$ so that they satisfy, in addition to $\left(\mathrm{Ra}^{\prime}\right),(\mathrm{Rb})$, and $(\mathrm{Rc})$, three more conditions:

(Rg) Each $K_{s}$ is of the form $H_{s}+\vec{u}_{s}$, where $H_{s} \in \mathcal{N}\left(K_{()}\right)$. Further, $\vec{u}_{()}=\overrightarrow{0}$.

(Rh) For each $s$ and each $L \in \mathcal{N}\left(K_{()}\right)$such that $L \subseteq H_{s}, K_{s \alpha}=L+\vec{u}_{s \alpha}$ for some $\alpha \in \mathfrak{c}$. Each $\vec{u}_{s \alpha}$ is of the form $\vec{u}_{s}+r_{s \alpha} \vec{e}_{s}$.

(Ri) For each $s$ and each non-empty relatively open $V \subseteq \widehat{K}_{s}$, there are uncountably many $\alpha$ such that $K_{s \alpha} \subseteq V$.

So, $(\mathrm{Rg})$ says that each $K_{s}$ is a translate of a subset of $K_{()}$. The $\widehat{K}_{s}$ are defined precisely as in $\S 2$, so that conditions $\left(\mathrm{Ra}^{\prime}\right),(\mathrm{Rb}),(\mathrm{Rc})$ already imply that $K_{s \alpha} \subseteq \widehat{K}_{s}$. Condition (Rg) guarantees that, unlike in $\S 2$, the projection $\pi_{0}: K_{t} \rightarrow K_{()}$is $1-1$ for each $t$ (and its inverse is translation by $\vec{u}_{t}$ ). Using Lemma 3.7, it is easy to see that conditions $\left(\mathrm{Ra}^{\prime}\right),(\mathrm{Rb}),(\mathrm{Rc}),(\mathrm{Rg}),(\mathrm{Rh})$, (Ri) can all be met.

If $f$ is a function on $L_{\omega}$ and $n=\operatorname{lh}(t)$, we shall say that $f$ is t-extensionconstant iff for all $\vec{x} \in K_{t}$ and all $\vec{y} \in L_{\omega} \cap \pi_{n}^{-1}\{\vec{x}\}, f(\vec{y})=f(\vec{x})$. By repeating the proof of the Note in the proof of Theorem 3.1, we see the following:

3.8. Lemma. If $N$ is metric and $f \in C\left(L_{\omega}, N\right)$, then $f$ is t-extensionconstant for all but countably many $t$.

In the next lemma, we use condition (Ri) to show that the $U_{t}$ form a $\pi$-base.

3.9. Lemma. If $V$ is open and non-empty in $L_{\omega}$, then for some $t, U_{t}$ $\subseteq V$

Proof. We may assume that $V=\left\{\vec{x} \in L_{\omega}: f(\vec{x}) \neq 0\right\}$, where $f \in$ $C\left(L_{\omega}, \mathbb{R}\right)$. First fix $s$ such that $V \cap \widehat{K}_{s}$ is non-empty, and then apply condition (Ri) plus Lemma 3.8 to set $t=s \alpha$, where $\alpha$ is chosen so that $K_{s \alpha} \subseteq V \cap \widehat{K}_{s}$ and $f$ is $s \alpha$-extension-constant. 
In the case of Theorem 3.1, all the $K_{t}$ were singletons, so " $t$-extensionconstant" meant "constant", and the instance of Lemma 3.9 used there was simple enough that we omitted the proof of it. In general, we cannot improve Lemma 3.8 to conclude that $f$ is constant on any open set. For example, the projection $\pi_{0}$ is 1-1 on each $K_{t}$, so cannot be constant on $K_{t}$ unless $K_{t}$ is a singleton. Applying Lemma 3.9, we get our last lemma.

3.10. Lemma. If $\mathcal{N}\left(K_{()}\right)$contains no singletons, then $\pi_{0} \in C\left(L_{\omega}, K_{()}\right)$ and $\Omega_{\pi_{0}}=\emptyset$.

Note, however, by condition $(\mathrm{Ri})$, that $\mathcal{N}\left(K_{()}\right)$contains no singletons iff no set in $\mathcal{N}\left(K_{()}\right)$has any isolated points. Of course, this is certainly true with $\mathcal{N}$ meaning "nowhere in $\mathcal{F}$ ". The specific features of this $\mathcal{N}$ appear in the conclusion of our proof.

Proof of Theorem 3.2(b). We fix an $M \notin \mathcal{F}$ and verify that for the space $X_{M}=L_{\omega}$ constructed above, $E_{0}\left(X_{M}, \mathbb{R}\right)=C\left(X_{M}, \mathbb{R}\right)$, but $E_{0}\left(X_{M}, M\right) \neq C\left(X_{M}, M\right)$. Since $X_{M}$ was constructed with $K_{()}$homeomorphic to a subset of $M$ which was nowhere in $\mathcal{F}, E_{0}\left(X_{M}, M\right) \neq C\left(X_{M}, M\right)$ follows from Lemma 3.10.

Now, fix $f \in C\left(X_{M}, \mathbb{R}\right)$. In view of Lemma 3.9, to prove that $f \in$ $E_{0}\left(X_{M}, \mathbb{R}\right)$, it suffices to fix an $s$ and find a non-empty open $V \subseteq U_{s}$ on which $f$ is constant. By Lemma 3.8, fix $\alpha$ such that $f$ is $s \alpha$-extension-constant. By condition (Rg), $K_{s \alpha}=H_{s \alpha}+\vec{u}_{s \alpha}$, where $H_{s \alpha} \in \mathcal{N}\left(K_{()}\right)$. Now, applying the properties of $\mathcal{N}$, we can choose an $L \in \mathcal{N}\left(K_{()}\right)$such that $L \subseteq H_{s \alpha}$ and $f$ is constant on $L+\vec{u}_{s \alpha}$. Applying condition (Rh) to $s \alpha$, we can choose a $\beta$ such that $K_{s \alpha \beta}=L+\vec{u}_{s \alpha \beta}$. So, let $V=U_{s \alpha \beta}$.

Finally, we show that the space $X_{M}$ can in fact be made independent of $M$. To do this, we use the next lemma to glue together the spaces constructed separately for each $M$.

3.11. Lemma. If $\left\{X_{\alpha}: \alpha<\mathfrak{c}\right\}$ is a collection of non-empty first countable uniform Eberlein compact spaces, then there is a first countable uniform Eberlein compact space $X$, with disjoint clopen subsets $J_{\alpha}$ homeomorphic to $X_{\alpha}$, such that $\bigcup_{\alpha \in \mathfrak{c}} J_{\alpha}$ is dense in $X$.

Proof. We may assume that each $X_{\alpha}$ is a weakly compact subset of the closed unit ball of the Hilbert space $\mathbb{B}_{0}$, and that $\mathbb{B}_{0}$ is a closed linear subspace of the Hilbert space $\mathbb{B}$, which contains unit vectors $\vec{m}_{\alpha}(\alpha \in \mathfrak{c})$ and $\vec{b}$, all orthogonal to each other and to $\mathbb{B}_{0}$.

Let $r_{\alpha}$, for $\alpha \in \mathfrak{c}$, enumerate $(0,1)$. Let $J_{\alpha}=X_{\alpha}+r_{\alpha} \vec{b}+\vec{m}_{\alpha}$. Then $J_{\alpha}$ is homeomorphic to $X_{\alpha}$ (via translation). Let $X$ be the union of the $J_{\alpha}$, together with all $r \vec{b}$ for $r \in[0,1]$. Then $X$ is norm bounded (by $\sqrt{3}$ ), and is weakly closed, since any limit of points in distinct $J_{\alpha}$ must be of the form 
$r \vec{b}$; the existence of these limits also shows that the union of the $J_{\alpha}$ is dense in $X$. The space $X$ is first countable by Lemma 2.3 . To see that the $J_{\alpha}$ are disjoint and (weakly) clopen in $X$, project along the $\vec{m}_{\alpha}$ direction.

We remark that translating along the $\vec{b}$ direction made $X$ first countable. Simply setting $J_{\alpha}=X_{\alpha}+\vec{m}_{\alpha}$ and letting $X$ be the union of the $J_{\alpha}$ plus $\{\overrightarrow{0}\}$ would make $X$ the non-first countable one-point compactification of the disjoint union of the $X_{\alpha}$. If there were $\kappa X_{\alpha}$, with $\kappa>\mathfrak{c}$, one could, of course, construct such a compactification $X$, but by Arkhangel'skili's Theorem, one could not produce a first countable space $X$ satisfying Lemma 3.11 (with $\mathfrak{c}$ replaced by $\kappa$ ).

3.12. Corollary. There is a single first countable uniform Eberlein compact space $X$ such that $E_{0}(X, \mathbb{R})=C(X, \mathbb{R})$, but $E_{0}(X, M) \neq C(X, M)$ for all compact metric $M \notin \mathcal{F}$.

Proof. Apply Lemma 3.11 and the fact that there are only $\mathfrak{c}$ compact metric spaces (up to homeomorphism).

4. General facts about $E_{0}(X, M)$. Here, we consider spaces $X$ for which $E_{0}(X, M)=C(X, M)$. This turns out to be an interesting topological property of $X$. We begin with a simple remark.

The condition $E_{0}(X, M)=C(X, M)$ is not hereditary to closed subsets of $X$, but it is, in many cases, hereditary to regular closed subsets - that is, to subsets of the form $\bar{U}$, where $U$ is open in $X$.

4.1. Lemma. Suppose that $X$ is a compact Hausdorff space, $E_{0}(X, \mathbb{R})$ $=C(X, \mathbb{R})$, and $Y$ is a regular closed subspace of $X$. Then $E_{0}(Y, \mathbb{R})=$ $C(Y, \mathbb{R})$.

Proof. Say $Y=\bar{U}$, where $U$ is open. Suppose $g \in C(Y, \mathbb{R})$. By the Tietze Extension Theorem, $g$ can be extended to an $f \in C(X, \mathbb{R})$. Then $\Omega_{g} \cap U=\Omega_{f} \cap U$. Since $E_{0}(X, \mathbb{R})=C(X, \mathbb{R})$, we find that $\Omega_{f}$ is dense in $X$, so $\Omega_{g}$ is dense in $Y$.

We remark that in Lemma 4.1, one can replace $\mathbb{R}$ by any Banach space (using a slightly longer proof), but not by an arbitrary metric space $M$. For a counter-example, let $M$ be a Cantor set and let $X$ be the cone over $M$. Then $E_{0}(X, M)=C(X, M)$ contains only constant functions. But $X$ contains a regular closed $Y$ homeomorphic to $M \times[0,1]$, and $E_{0}(Y, M) \neq C(Y, M)$. Also, even in the simple case $M=\mathbb{R}$, the property $E_{0}(Y, \mathbb{R})=C(Y, \mathbb{R})$ holds for all closed $Y \subseteq X$ iff $X$ is scattered; if $X$ is not scattered, then $X$ will contain a closed subset $Y$ which is separable with no isolated points, which implies $E_{0}(Y, \mathbb{R}) \neq C(Y, \mathbb{R})$ (by $(2) \Rightarrow(1)$ of Theorem 4.2 below).

Now, to study the property $E_{0}(X, M)=C(X, M)$, it is convenient to generalize our notions in two ways. 
First, although $X$ will always be compact and $M$ will always be metric, we look at more general functions from $X$ into $M$. In particular, recall that $f: X \rightarrow M$ is called Borel measurable iff the inverse image of every open set is a Borel subset of $X$, and Baire measurable iff the inverse image of every open set is a Baire subset of $X$; the Baire sets are the $\sigma$-algebra generated by the open $F_{\sigma}$-sets. The Baire measurable functions into a separable Banach space form the least class of functions containing the continuous functions and closed under pointwise limits.

Second, we consider also $\widehat{\Omega}_{f}$, which we define to be the union of all open $U \subseteq X$ such that for some first category set $C \subseteq X, f$ is constant on $U \backslash C$. Note that regardless of $f, \Omega_{f}$ (defined in the introduction) and $\widehat{\Omega}_{f}$ are open, with $\Omega_{f} \subseteq \widehat{\Omega}_{f}$. If $f$ is continuous, then $\Omega_{f}=\widehat{\Omega}_{f}$.

The property $E_{0}(X, \mathbb{R})=C(X, \mathbb{R})$ is just one of a sequence of related properties:

(1) Every non-empty open subset of $X$ is either non-separable or contains an isolated point.

(2) $E_{0}(X, \mathbb{R})=C(X, \mathbb{R})$.

(3) For all metric spaces $M, E_{0}(X, M)=C(X, M)$.

$\left(3^{\prime}\right)$ For all separable metric spaces $M$ and Baire measurable $f: X \rightarrow M$, $\widehat{\Omega}_{f}$ is dense in $X$.

$\left(3^{\prime \prime}\right)$ For all separable metric spaces $M$ and Baire measurable $f: X \rightarrow M$, $\Omega_{f}$ is dense in $X$.

(4) For all separable metric spaces $M$ and Borel measurable $f: X \rightarrow M$, $\widehat{\Omega}_{f}$ is dense in $X$.

(5) For all separable metric spaces $M$ and Borel measurable $f: X \rightarrow M$, $\Omega_{f}$ is dense in $X$.

(6) In $X$, every non-empty $G_{\delta}$-set has a non-empty interior.

$(*)$ In $X$, every first category set is nowhere dense.

Conditions (1)-(6) are listed in order of increasing strength. Condition $(*)$ does not fit into the sequence, but is relevant by the next theorem.

4.2. TheOrem. Suppose $X$ is compact Hausdorff. Then

$$
(6) \Rightarrow(5) \Rightarrow(4) \Rightarrow(3) \Leftrightarrow\left(3^{\prime}\right) \Leftrightarrow\left(3^{\prime \prime}\right) \Rightarrow(2) \Rightarrow(1) .
$$

Furthermore, (5) is equivalent to $(*)$ plus (4).

Proof. For $(2) \Rightarrow(1)$, assume (1) fails; so there is a non-empty open $U$ which is separable and has no isolated points. Let $Y=\bar{U}$. By Lemma 4.1, it is sufficient to produce an $f \in C(Y, \mathbb{R}) \backslash E_{0}(Y, \mathbb{R})$. Let $\left\{p_{n}: n \in \omega\right\}$ be dense in $U$, hence in $Y$. For each distinct $m, n,\left\{f \in C(Y, \mathbb{R}): f\left(p_{m}\right) \neq\right.$ $\left.f\left(p_{n}\right)\right\}$ is dense and open in $C(Y, \mathbb{R})$ (in the usual norm topology), so by the Baire Category Theorem, there is an $f \in C(Y, \mathbb{R})$ such that $f\left(p_{m}\right) \neq f\left(p_{n}\right)$ 
whenever $m \neq n$. But then for each $r \in \mathbb{R}, f^{-1}\{r\}$ contains at most one $p_{n}$, and is hence nowhere dense in $Y$, since $Y$ has no isolated points. Thus, $f \notin E_{0}(Y, \mathbb{R})$.

Clearly, $\left(3^{\prime \prime}\right) \Rightarrow\left(3^{\prime}\right) \Rightarrow(3)$, so to prove these three are equivalent, we assume (3), fix a Baire measurable $f: X \rightarrow M$, and show that $\Omega_{f}$ is dense in $X$. Since $M$ can be embedded into a separable Banach space, we may assume that $M$ is a Banach space; now, we can let $g_{n}: X \rightarrow M$, for $n \in \omega$, be continuous functions such that $f$ can be obtained from the $g_{n}$ by some transfinite iteration of taking pointwise limits. Define $g: X \rightarrow M^{\omega}$ by $g(x)_{n}=g_{n}(x)$. Then $\Omega_{g} \subseteq \Omega_{f}$, and, by (3), $\Omega_{g}$ is dense in $X$.

To prove $(6) \Rightarrow(5)$, observe that for any compact $X$, if $H$ is a non-empty closed $G_{\delta}$ and $f$ is a Borel measurable map into a second countable space, there is always a non-empty closed $G_{\delta}$-set $K \subseteq H$ such that $f$ is constant on $K$.

The rest of the chain of implications from (6) down to (1) are now trivial. To see that $(5) \Rightarrow(*)$, let $C$ be first category; then $C \subseteq \bigcup_{n \in \omega} K_{n}$, where each $K_{n}$ is closed nowhere dense. Define $f: X \rightarrow 2^{\omega}$ so that $f(x)_{n}$ is 1 if $x \in K_{n}$ and 0 if $x \notin K_{n}$. Then $\Omega_{f}$ is dense and open, and is disjoint from all the $K_{n}$, so $C$ is nowhere dense.

To see that $(*)$ plus (4) implies (5), we let $f$ be Borel measurable; to prove $\Omega_{f}$ dense, we fix a non-empty open $V$ and try to find a non-empty open $U \subseteq V$ such that $f$ is constant on $U$. By (4), there is a non-empty open $W \subseteq V$ such that $f$ is constant on $W \backslash C$ for some first category $C$. By $(*), C$ is nowhere dense, so let $U=W \backslash \bar{C}$.

A familiar example of a space satisfying (6) is $\beta \mathbb{N} \backslash \mathbb{N}$.

Conditions (5), (4), $\left(3^{\prime \prime}\right)$, and $\left(3^{\prime}\right)$ involve arbitrary Baire or Borel measurable maps. Each of these conditions is equivalent to the restatement we obtain by replacing $M$ by the Cantor set $2^{\omega}$. This is easily seen by translating the condition to one involving an $\omega$-sequence of Borel or Baire sets. For example, (5) is equivalent to the statement that given Borel sets $B_{n}$ $(n \in \omega)$, the union of all open $U$ such that for all $n, U \subseteq B_{n}$ or $U \cap B_{n}=\emptyset$, is dense in $X$.

This is not true for (3), which involves continuous functions. For example, if $X$ is connected, then, trivially, $E_{0}\left(X, 2^{\omega}\right)=C\left(X, 2^{\omega}\right)$, whereas $E_{0}(X, \mathbb{R})$ need not be all of $C(X, \mathbb{R})$. If $X$ is zero-dimensional, then $E_{0}\left(X, 2^{\omega}\right)=$ $C\left(X, 2^{\omega}\right)$ does imply (3). In fact, for zero-dimensional spaces, (3) has a restatement in terms of sequences of clopen sets (see the proof of Theorem 4.3(c) below).

Regarding $(3) \Rightarrow(2)$, if $E_{0}(X, M)=C(X, M)$ for any $M$ containing an interval, then (2) holds. By Theorem 3.2, (2) does not imply (3), although it is easy to see that (2) implies $E_{0}\left(X, \mathbb{R}^{n}\right)=C\left(X, \mathbb{R}^{n}\right)$ for each finite $n$. 
Counter-examples to the other implications of Theorem 4.2 reversing are provided by some fairly familiar spaces, as we point out below. However, the implications do reverse for certain families of spaces. In particular, we consider the cases when $X$ is extremally disconnected, when $X$ is an Eberlein compact space, when $X$ is a LOTS, and when $X$ has the $c c c . X$ is called extremally disconnected iff the closure of every open subset of $X$ is clopen. $X$ is an Eberlein compact space iff $X$ is homeomorphic to a weakly compact subspace of a Banach space. $X$ is a LOTS iff $X$ is a totally ordered set, given the order topology. $X$ has the ccc iff there is no uncountable family of disjoint open sets in $X$.

The following theorem summarizes what we know for these and some other simple classes.

\subsection{Theorem. Let $X$ be compact Hausdorff.}

(a) If $X$ is metric, then $(1) \Leftrightarrow(5)$, and (1)-(5) hold iff the isolated points of $X$ are dense in $X$.

(b) If $X$ is extremally disconnected, then $(2) \Leftrightarrow(4)$.

(c) If $X$ is zero-dimensional, then $(2) \Leftrightarrow(3)$.

(d) If $X$ is ccc, then $(3) \Leftrightarrow(5)$.

(e) If $X$ is an Eberlein compact space, then $(4) \Leftrightarrow(5)$, and (4)-(5) hold iff the isolated points of $X$ are dense in $X$.

(f) If $X$ is a LOTS, then $(1) \Leftrightarrow(3)$.

Proof. (a) is immediate from the fact that compact metric spaces are separable.

For (b), assume (2), and let $f: X \rightarrow M$ be Borel measurable. Let $\left\{B_{n}: n \in \omega\right\}$ be an open base for $M$. Since each $f^{-1}\left(B_{n}\right)$ is a Borel set, there are open $U_{i} \subseteq X$, for $i \in \omega$, such that each $f^{-1}\left(B_{n}\right)$ is in the $\sigma$-algebra generated by $\left\{U_{i}: i \in \omega\right\}$. Let $K_{i}=\bar{U}_{i}$, which is clopen. Define $g: X \rightarrow 2^{\omega}$ so that $g(x)_{i}=1$ iff $x \in K_{i}$. Since $2^{\omega}$ is embeddable in $\mathbb{R},(2)$ implies that $\Omega_{g}$ is dense. Since $\bigcup_{i \in \omega}\left(K_{i} \backslash U_{i}\right)$ is first category, $\Omega_{g} \subseteq \widehat{\Omega}_{f}$, so $\widehat{\Omega}_{f}$ is dense.

For (c), assume (2), and let $f: X \rightarrow M$ be continuous. Let the $B_{n}$ be as in the proof of (b). Since each $f^{-1}\left(B_{n}\right)$ is an open $F_{\sigma}$-set, there are clopen sets $K_{i} \subseteq X$ for $i \in \omega$ such that each $f^{-1}\left(B_{n}\right)$ is a union of some subfamily of the $K_{i}$. Now, construct $g$ as in the proof of (b), and note that $\Omega_{g} \subseteq \Omega_{f}$.

For (d), assume $\left(3^{\prime \prime}\right)$, and let $f: X \rightarrow M$ be Borel measurable. Since $X$ is ccc, there is a Baire measurable $g: X \rightarrow M$ and a Baire first category set $C$ such that $f(x)=g(x)$ for all $x \notin C$. Define $h: X \rightarrow M \times\{0,1\}$ so that $h(x)=(g(x), 0)$ if $x \notin C$, and $h(x)=(g(x), 1)$ if $x \in C$. Then, applying $\left(3^{\prime \prime}\right)$ to $h, \Omega_{h}$ is dense in $X$. Since $\Omega_{h} \subseteq \Omega_{g}$ and $\Omega_{h} \cap C=\emptyset, \Omega_{f}$ is dense in $X$.

For (e), assume that $X$ is an Eberlein compact space and satisfies (4); we prove that the isolated points are dense. By a result of Benyamini, Rudin, and Wage [4], there is a dense $G_{\delta}$-set $Y \subseteq X$ such that $Y$ is metrizable 
in its relative topology. Fix some metric on $Y$; then for $E \subseteq Y$, $\operatorname{diam}(E)$ denotes the diameter of $E$ with respect to this metric. For each $n$, let $\mathcal{W}_{n}$ be a maximal disjoint family of open non-empty subsets of $Y$ of diameter $\leq 2^{-n}$; then $W_{n}=\bigcup\left\{W: W \in \mathcal{W}_{n}\right\}$ is open and dense. Assume also that each $\mathcal{W}_{n+1}$ refines $\mathcal{W}_{n}$ in the sense that for all $W \in \mathcal{W}_{n+1}$ there is $V \in \mathcal{W}_{n}$ such that $W \subseteq V$, and for each $V \in \mathcal{W}_{n}$ which is not a singleton, there are at least two $W \in \mathcal{W}_{n+1}$ such that $W \subseteq V$. Let $Z=\bigcap_{n} W_{n}$; then $Z$ is also a dense $G_{\delta}$-subset of $X$. For each $n$, let $f_{n}: Z \rightarrow 2$ be any function such that $f_{n}$ is constant on every $W \in \mathcal{W}_{n+1}$ and $f_{n}$ is constant on no $V \in \mathcal{W}_{n}$ unless $V$ is a singleton. This defines $f: Z \rightarrow 2^{\omega}$ by $f(z)_{n}=f_{n}(z)$. Let $M$ be the disjoint sum of $2^{\omega}$ and a single point, $p$, and extend $f$ to a function $\widetilde{f}: X \rightarrow M$ by mapping $X \backslash Z$ to $p$. Then $\widetilde{f}$ is Borel measurable, and every point in $\widehat{\Omega}_{\tilde{f}}$ is isolated in $X$.

For (f), assume (1), and fix $f \in C(X, M)$; we must show that $\Omega_{f}$ is dense. So, fix a non-empty open interval $(a, b) \subseteq X$. We must produce a non-empty open $W \subseteq(a, b)$ such that $f$ is constant on $W$. This is trivial if $(a, b)$ contains an isolated point, so assume that $(a, b)$ contains no isolated points, and hence is non-separable. For each $n$, there is a finite cover of $[a, b]$ by open intervals, $I_{1}^{n}, I_{2}^{n}, \ldots$, such that each $\operatorname{diam}\left(f\left(I_{j}^{n}\right)\right) \leq 1 / n$. Since $(a, b)$ is non-separable, we can choose $W \subseteq(a, b)$ to be an open interval which contains none of the endpoints of any $I_{j}^{n}$. Then for each $n$, $W$ is a subset of some $I_{j}^{n}$, so $\operatorname{diam}(f(W)) \leq 1 / n$. Thus, $f$ is constant on $W$.

Now, to see that most of the implications of Theorem 4.3 are as strong as possible, we look at several examples, and we include two useful vehicles for examining properties of topological spaces $X$ : the Boolean algebra of regular open subsets of $X$, and the corresponding notion of forcing.

In general, for a LOTS, (1) need not imply (4). A simple counter-example is $X=[0,1]^{\omega}$, ordered lexically; (4) is refuted by $f(x)=\sum_{n \in \omega} x_{n} \cdot 2^{-n}$. One can replace $[0,1]$ by the Cantor set here to get a zero-dimensional LOTS, providing also a counter-example to (c) extending to $(2) \Leftrightarrow(4)$.

If $X$ is an Eberlein compact space, then by (e), conditions (4) or (5) can hold only in the trivial case that the isolated points of $X$ are dense in $X$; it is easy to see that (6) holds iff $X$ is finite. Thus, only (1), (2), and (3) are of interest, and for these, the Eberlein compact spaces can be tailored to satisfy whatever we want. The one we constructed in $\S 2$ satisfied (1), but not (2), whereas $\S 3$ produced Eberlein compact spaces which satisfied (3) but not (4), and (2) but not (3).

The Stone space of an atomless probability algebra is a compact extremally disconnected space which satisfies (1) but not (3). To refute (3), let the $K_{i}(i \in \omega)$ be clopen independent events of probability $1 / 2$, and con- 
struct $g$ as in the proof of Theorem 4.3(b). This provides a counter-example to replacing (2) by (1) in either (b) or (c).

Each of the conditions (4), (*), and (5) is equivalent to an algebraic property of the Boolean algebra of regular open subsets of $X$ (see $[9,16]$ ). Hence, each of these three conditions holds for $X$ iff it holds for the absolute (or projective cover) of $X$. Specifically, condition (4) is equivalent to the $(\omega, \omega)$-distributive law:

$$
\bigwedge_{n \in \omega} \bigvee_{i \in \omega} b_{n, i}=\bigvee\left\{\bigwedge_{n \in \omega} b_{n, \varphi(n)}: \varphi \in \omega^{\omega}\right\}
$$

Condition $(*)$ is equivalent to the weak $(\omega, \infty)$-distributive law: for each cardinal $\kappa$,

$$
\bigwedge_{n \in \omega} \bigvee_{\alpha \in \kappa} b_{n, \alpha}=\bigvee\left\{\bigwedge_{n \in \omega} \bigvee_{\alpha \in \varphi(n)} b_{n, \alpha}: \varphi \in\left([\kappa]^{<\omega}\right)^{\omega}\right\} .
$$

Here, $[\kappa]^{<\omega}$ is the set of finite subsets of $\kappa$. By Theorem 4.2, condition (5) is simply (4) plus $(*)$, and is hence equivalent to the standard $(\omega, \infty)$ distributive law.

A (compact) Suslin line in which every open interval is non-separable is a ccc LOTS (with no isolated points) which satisfies (1), and hence (5), applying (d) and (f) of the Theorem. Of course, the Suslin line does not satisfy (6). The absolute of a Suslin line is a compact ccc extremally disconnected space which satisfies (5) but not (6). So is $\beta \mathbb{N}$, but this example is "trivial" because the isolated points are dense. Note, however, that it is consistent with the axioms of set theory that there are no Suslin lines. In the absence of Suslin lines, (5) for a ccc space would imply that the isolated points are dense, because the existence of a Suslin line is equivalent to the existence of a Suslin algebra (a non-atomic ccc $(\omega, \infty)$-distributive complete Boolean algebra).

We may regard the open (or regular open) subsets of $X$ as a forcing order (see a set theory text, such as [10] or [11]). Then (4) is simply the statement that the order adds no reals, while (5) is the stronger statement that the order adds no $\omega$-sequences. Condition $(*)$ is the finite approximation property familiar from random real forcing or Sacks forcing: for each $\kappa$ and each $\psi: \omega \rightarrow \kappa$ in the generic extension, there is a $\varphi: \omega \rightarrow[\kappa]^{<\omega}$ in the ground model such that each $\psi(n) \in \varphi(n)$. Prikry forcing at a measurable cardinal (see $\S 37$ of [10]) is an example of a forcing order (and hence, by the standard translation, a compact extremally disconnected space) which satisfies (4) but not $(*)$, and hence not (5). Another such example is Namba forcing (see $\S 26$ of [10]).

Finally, it is natural to ask which of the properties $(1)-(6),(*)$ are preserved by finite products. Now, (1) and (6) are, trivially. We do not know 
about (2), but (3) is; to see this, identify $C(X \times Y, M)$ with $C(X, C(Y, M))$, and note that $C(Y, M)$ is another metric space. Property $(5)=(4)+(*)$ is refuted by a well-known forcing order: Let $S \subset \omega_{1}$ be stationary and co-stationary. Let $\mathbb{P}$ and $\mathbb{Q}$ be Jensen's forcings for shooting a club through $S$ and $\omega_{1} \backslash S$, respectively (see VII.H25 of [11]). Then $\mathbb{P}, \mathbb{Q}$ each satisfy (5), while $\mathbb{P} \times \mathbb{Q}$ collapses $\omega_{1}$, and hence satisfies neither (4) nor $(*)$. One may now translate $\mathbb{P}, \mathbb{Q}$ into compact extremally disconnected spaces (by the standard translation), or into Corson compact spaces (using the fact that these partial orders have no decreasing $\omega_{1}$ chains).

Preservation by infinite products is uninteresting. If $X$ is an infinite product of spaces with more than one point, then all of (2)-(6) fail, as does (*), whereas (1) will hold if, for example, infinitely many of the $X_{n}$ are non-separable. See Theorem 5.5.2 for more about such products.

Some of the results in this section overlap results of Bella, Hager, Martinez, Woodward, and Zhou $[2,3,12]$. They also defined $E_{0}(X, \mathbb{R})$ (which they called $d c(X)$ ), and they considered spaces with our property (2), which they called DC-spaces. With somewhat different terminology, they proved what amounts to the fact that (6) implies (2), and that (1) and (2) are equivalent when $X$ is a LOTS.

5. On Banach spaces. In this section, we make a few remarks on $E_{0}(X, M)$ in the case that $X$ is an arbitrary compact Hausdorff space and $M$ is a Banach space. For definiteness, we take the scalar field to be $\mathbb{R}$, but all the results are unchanged if we replace $\mathbb{R}$ by $\mathbb{C}$.

5.1. Types of $X$. As we have seen in $\S 4$, there are many $X$ for which $E_{0}(X, M)=C(X, M)$. For a given $X$, this can depend on $M$. However, the range of each function in $C(X, M)$ is compact, and hence embeddable in the Hilbert cube, which in turn is embeddable in every infinite-dimensional Banach space. It follows that there are really only three possibilities:

(1) $E_{0}(X, M)=C(X, M)$ for all Banach spaces $M$.

(2) $E_{0}(X, M)=C(X, M)$ for all finite-dimensional $M$, but not for any infinite-dimensional $M$.

(3) $E_{0}(X, M) \neq C(X, M)$ for all Banach spaces $M$.

Furthermore, there are Eberlein compact spaces with no isolated points realizing each of these possibilities ((3) is trivial; see $\S 3$ for $(1)$ and $(2))$.

5.2. Abstract properties of $E_{0}$. In studying the properties of $E_{0}(X, M)$ as a normed linear space, we can isolate the two properties which are of fundamental importance. If $f, g_{1}, g_{2} \in C(X, M)$, let us say that $f$ is refined by $g_{1}, g_{2}$ iff for all $x, y \in X$, if $g_{1}(x)=g_{1}(y)$ and $g_{2}(x)=g_{2}(y)$ then $f(x)=f(y)$. A linear subspace $E \subseteq C(X, M)$ has the refinement property iff 
for all $f, g_{1}, g_{2} \in C(X, M)$, if $g_{1}, g_{2} \in E$ and $f$ is refined by $g_{1}, g_{2}$, then $f \in E$. We say that $E$ has the disjoint summation property iff whenever $\sum_{i \in \omega} f_{i}=f$ in $C(X, M)$, each $f_{i} \in E$, and the sets $\left\{x: f_{i}(x) \neq 0\right\}$, for $i \in \omega$, are all disjoint, then $f \in E$. The set of polynomial functions in $C([0,1], \mathbb{R})$ has the disjoint summation property (trivially) but not the refinement property, while the set of functions which are constant in some neighborhood of $1 / 2$ has the refinement property but not the disjoint summation property. Let us call $E$ a nice subspace of $C(X, M)$ iff $E$ has both properties. Examples of nice $E$ are $E_{0}(X, M), C(X, M)$, and the space of all constant functions. Or, one may fix any open $U \subseteq X$; then $\left\{f \in C(X, M): U \subseteq \bar{\Omega}_{f}\right\}$ is nice. Another example is the functions of essentially countable range; that is, let $\mu$ be a Baire measure on $X$, and then let $D(X, M, \mu)$ be the set of $f \in C(X, M)$ such that for some $\mu$-null-set $S \subseteq X, f(X \backslash S)$ is countable. Another is the category analog of this - the set of $f \in C(X, M)$ such that for some countable $P \subset M, \bigcup\left\{\operatorname{int}\left(f^{-1}\{p\}\right): p \in P\right\}$ is dense in $X$ (int denotes interior).

One advantage of studying nice $E$ is that we may restrict our attention to the case where $E$ separates the points of $X$. In general, given $E \subseteq C(X, M)$, we may define an equivalence relation $\sim$ on $X$ by $x \sim y$ iff $f(x)=f(y)$ for all $f \in E$. Let $Y$ be the quotient, $X / \sim$; then $Y$ is a compact Hausdorff space, and there is a canonical projection, $\pi$, from $Y$ onto $X$. Let $E^{\prime}=$ $\{g \in C(Y, M): g \circ \pi \in E\}$. Then $E^{\prime}$ is isometric to $E$, and $E^{\prime}$ separates the points of $Y$. Further, both the refinement property and the disjoint summation property are preserved here, so if $E$ is nice, then so is $E^{\prime}$.

Some examples, when we start with $E=E_{0}(X, M)$ : For the spaces constructed in $\S 2$ : If $X=L_{\omega}$, then $Y$ is a singleton. If $X=L_{2}$, then $Y$ is obtained by collapsing $L_{1}$ to a point. In these two cases, $E^{\prime}=E_{0}(Y, M)$, but this is not in general true. For example, let $Q$ be any dense subset of $[0,1]$, and form $X$ by attaching a copy of the $L_{\omega}$ of $\S 2$ to each $q \in Q$, where each copy goes off in some perpendicular direction. There is then a natural retraction, $r: X \rightarrow[0,1]$, and $E_{0}(X, \mathbb{R})$ consists of all functions of the form $f \circ r$, where $f \in C([0,1], \mathbb{R})$. So, we may identify $Y$ with $[0,1]$ and $\pi$ with $r$, and $E^{\prime}$ is $C(Y, \mathbb{R})$, not $E_{0}(Y, \mathbb{R})$.

5.3. The refinement property. Suppose that $E \subseteq C(X, M)$ has the refinement property. If $\varphi \in C(M, M)$ and $f \in E$, then $\varphi \circ f \in E$ (since $\varphi \circ f$ is refined by $f, f)$. If $M=\mathbb{R}$, and we view $C(X, M)$ as a Banach algebra (under pointwise multiplication), then $E$ is a subalgebra. More generally, if we fix any non-zero vector $\vec{v} \in M$, we may let $\widehat{E} \subseteq C(X, \mathbb{R})$ be the set of all $g \in C(X, \mathbb{R})$ such that the map $x \mapsto g(x) \vec{v}$ is in $E$. Note that this does not depend on the $\vec{v}$ chosen, and if $g \in \widehat{E}$ and $f \in E$, then $g f \in E$. Note also that $\widehat{E}$ is nice. 
It follows that if $E \subseteq C(X, M)$ has the refinement property and separates the points of $X$, then $E$ is dense in $C(X, M)$. To see this, fix $f \in C(X, M)$. If $M=\mathbb{R}^{n}$, just apply the Stone-Weierstrass Theorem to $f$ composed with the projections onto $n$ one-dimensional subspaces. Then, for a general $M$, first approximate $f$ arbitrarily closely by a map into a finite-dimensional subspace.

Actually, one can get more than just what is provided by a simple application of the Stone-Weierstrass Theorem. For example, we can arrange for the approximating function to be identically zero wherever $f$ is zero:

5.3.1. Lemma. Suppose $E \subseteq C(X, M)$ has the refinement property and separates the points of $X$. Fix $f \in C(X, M)$ and fix $\varepsilon>0$. Then there is a $g \in E$ with $\|g-f\| \leq \varepsilon,\|g\|=\|f\|$, and $\|g(x)\|=\|f(x)\|$ for all $x$ such that $\|f(x)\|$ equals either 0 or $\|f\|$.

Proof. Assume $\varepsilon<\|f\|$. Fix $h \in E$ with $\|h-f\| \leq \varepsilon / 2$. Then, let $\varphi$ : $M \rightarrow M$ be any continuous map such that for all $\vec{v} \in M,\|\varphi(\vec{v})-\vec{v}\| \leq \varepsilon / 2$, $\varphi(\vec{v})=\overrightarrow{0}$ when $\|\vec{v}\| \leq \varepsilon / 2$, and $\varphi(\vec{v})=\|f\|$ when $|\|\vec{v}\|-\|f\|| \leq \varepsilon / 2(\varphi$ can just move each $\vec{v}$ radially). Then, let $g=\varphi \circ h$.

5.4. Is $E_{0}(X, M)$ a Banach space? Certainly it is in the extreme cases where it is all of $C(X, M)$ and where it contains only the constant functions. To analyze the general situation, we may, as pointed out above, just consider the case where $E \subseteq C(X, M)$ is nice and separates the points of $X$. Then, clearly, $E$ is a Banach space in the standard norm iff it is all of $C(X, M)$. Furthermore, if $E$ is not all of $C(X, M)$, then, following Bernard and Sidney $[6,15]$, it is not even Banachizable; that is, there is no norm which makes $E$ into a Banach space and gives $E$ a topology finer than the one inherited from $C(X, M)$. In fact, every nice $E$ is barreled, which is a stronger property. There are a number of equivalents to being barreled, discussed in [15]. One is that for every linear space $\mathbb{L}$ with $E \subseteq \mathbb{L} \varsubsetneqq \bar{E}, \mathbb{L}$ is not Banachizable ( $\bar{E}$ is the completion of $E$; here, $\bar{E}=C(X, M)$ ). Another is the "weak sequential property" for $E$, which is the conclusion of the next lemma; this is a convenient way of establishing barreledness. The proof of the next lemma is very similar in spirit to that of Theorem 2 of [15], but we include it because at first sight, the proof as stated in [15] might appear to require some additional assumptions about $E$ and $X$. The two examples above of subspaces of $C([0,1], \mathbb{R})$ show that neither of the two components of "nice", "refinement property", and "disjoint summation property" is sufficient here.

5.4.1. Lemma. Let $X$ be compact and let $M$ be a Banach space. Suppose that $E$ is a nice subspace of $C(X, M)$. Let $\Lambda_{n}$, for $n \in \omega$, be in the dual space, $E^{*}$. Assume that for every $g \in E, \Lambda_{n}(g) \rightarrow 0$. Then $\sup _{n}\left\|\Lambda_{n}\right\|<\infty$. 
Proof. As pointed out above, we may also assume that $E$ is dense in $C(X, M)$, so we may consider $\Lambda_{n}$ to be in $C(X, M)^{*}$. Note that if $E=$ $C(X, M)$, the conclusion is immediate by the Banach-Steinhaus Theorem. In any case, whenever $\mathbb{H}$ is a closed linear subspace of $C(X, M)$ such that $\mathbb{H} \subseteq E$,

$$
\sup \left\{\left|\Lambda_{n}(h)\right|: h \in \mathbb{H} \cap \bar{B}(0,1) \& n \in \omega\right\}<\infty .
$$

Here, $\bar{B}(0,1)$ is the closed unit ball of $C(X, M)$. Now, assume that $\sup _{n}\left\|\Lambda_{n}\right\|$ $=\infty$. We shall get a contradiction by applying (1).

For any $f \in C(X, M)$, let $\operatorname{supt}(f)$ be the closure of $\{x \in X: f(x) \neq \overrightarrow{0}\}$. By compactness of $X$, we may fix a point $p$ such that for all neighborhoods $V$ of $p$,

$$
\sup \left\{\left|\Lambda_{n}(f)\right|: f \in \bar{B}(0,1) \& n \in \omega \& \operatorname{supt}(f) \subseteq V\right\}=\infty .
$$

By Lemma 5.3.1 (applied to $\widehat{E}$ - see above), let $g \in \widehat{E}$ be such that $\|g\|=1$, $g(p)=1$, and $\operatorname{supt}(g) \subseteq V$. Then $\mathbb{H}=\{g \vec{v}: \vec{v} \in M\}$ is a closed linear subspace of $C(X, M)$ (isometric to $M$ ) such that $\mathbb{H} \subseteq E$, so we may apply (1) above. It follows, by considering functions of the form $x \mapsto f(x)-g(x) f(p)$, that for all neighborhoods $V$ of $p$,

$$
\sup \left\{\left|\Lambda_{n}(f)\right|: f \in \bar{B}(0,1) \& n \in \omega \& \operatorname{supt}(f) \subseteq V \& f(p)=\overrightarrow{0}\right\}=\infty .
$$

Next, we show that for all neighborhoods $V$ of $p$,

$$
\sup \left\{\left|\Lambda_{n}(g)\right|: g \in \bar{B}(0,1) \cap E \& n \in \omega \& \operatorname{supt}(g) \subseteq V \backslash\{p\}\right\}=\infty .
$$

To see this, fix $K>0$, and then fix $n$ and $f \in C(X, M)$ such that $\|f\| \leq 1$, $\operatorname{supt}(f) \subseteq V, f(p)=\overrightarrow{0}$, and $\left|\Lambda_{n}(f)\right| \geq 3 K$. Let $f^{\prime} \in C(X, M)$ be such that $\left\|f^{\prime}\right\| \leq 1, \operatorname{supt}\left(f^{\prime}\right) \subseteq V, f^{\prime}$ vanishes in some neighborhood of $p$, and $\left\|f^{\prime}-f\right\| \leq K /\left\|\Lambda_{n}\right\|$. Applying Lemma 5.3.1 to $f^{\prime}$, let $g \in E$ be such that $\|g\| \leq 1, \operatorname{supt}(g) \subseteq V, g$ vanishes in some neighborhood of $p$, and $\left\|g-f^{\prime}\right\| \leq$ $K /\left\|\Lambda_{n}\right\|$. Then $\left|\Lambda_{n}(g)\right| \geq K$.

Thus, we may inductively choose open neighborhoods $V_{j}$ of $p, n_{j} \in \omega$, and $h_{j} \in E$ such that each $\bar{V}_{j+1} \subseteq V_{j}$, supt $\left(h_{j}\right) \subseteq V_{j} \backslash \bar{V}_{j+1},\left\|h_{j}\right\|=1$, and $\left|\Lambda_{n_{j}}\left(h_{j}\right)\right| \geq j$. Let $\mathbb{H}$ be the closed linear span in $C(X, M)$ of the $h_{j}$. Since the $h_{j}$ are disjointly supported, it follows that $\mathbb{H} \subseteq E$ (and $\mathbb{H}$ is isometric to $\left.c_{0}\right)$, so we have a contradiction to (1) above.

5.5. Is $E_{0}(X, M)$ first category in itself? We ask this because if $E_{0}(X, M)$ is of second category, then Lemma 5.4 .1 becomes trivial by the Banach-Steinhaus Theorem. Fortunately, $E_{0}(X, M)$ is first category in many cases; for example, when $X$ contains a non-empty separable open subset with no isolated points (see the proof of $(2) \Rightarrow(1)$ of Theorem 4.2). In fact, as pointed out by Bernard and Sidney, the original interest of $E_{0}(X)$ was that it provided examples of first category normed linear spaces which satisfy the Banach-Steinhaus Theorem, as well as a number of other results 
usually proved by category arguments. The following lemma describes some other situations in which $E_{0}(X, M)$ is of first category.

5.5.1. Lemma. Let $X$ be compact and let $M$ be a Banach space. Suppose that $E_{0}(X, M)$ is not a Banach space. Then $E_{0}(X, M)$ is of first category in itself if either of the following holds:

(a) $M$ is infinite-dimensional.

(b) $X$ is zero-dimensional.

Pr o o f. First, as indicated above, we may pass to a quotient and consider a nice $E \subseteq C(X, M)$ which is dense in $C(X, M)$ but is not all of $C(X, M)$; of course, in (b), this quotient operation is trivial. Now, we need only show that $E$ is of first category in $C(X, M)$.

Whenever $H$ and $K$ are closed subsets of $X$, let $U(H, K)=\{g \in$ $C(X, M): g(H) \cap g(K)=\emptyset$. Note that $U(H, K)$ is always open in $C(X, M)$. If $H$ and $K$ are disjoint, then either (a) or (b) guarantees that $U(H, K)$ is dense in $C(X, M)$.

Fix an $f \in C(X, M) \backslash E$. Since $f(X)$ is second countable, there are closed $H_{n}, K_{n} \subseteq X$ for $n \in \omega$ such that each $H_{n} \cap K_{n}=\emptyset$, and for all $x, y \in X$, if $f(x) \neq f(y)$, then for some $n, x \in H_{n}$ and $y \in K_{n}$. Let $G=\bigcap_{n \in \omega} U\left(H_{n}, K_{n}\right)$. Then $G$ is a dense $G_{\delta}$, and $f$ is refined by $g, g$ for all $g \in G$, so $G$ is disjoint from $E$.

The situation for finite-dimensional $M$ seems more complicated. We do not actually have an example of an $E_{0}(X, \mathbb{R})$ which is second category but not a Banach space, although it is easy to produce a consistent example of this by forcing $[10,11]$. In the ground model, $V$, let $X=L_{\omega}$ be the space constructed in the proof of Theorem $3.2(\mathrm{~b})$, so $E_{0}(X, \mathbb{R})=C(X, \mathbb{R})$. Let $V[G]$ add one Cohen real. Then, in $V[G], E_{0}(X, \mathbb{R})$ is of second category, since it contains the ground model $C(X, \mathbb{R})$, which is of second category with this forcing. However, in $V[G], E_{0}(X, \mathbb{R})$ is not all of $C(X, \mathbb{R})$, since $V[G]$ will contain a $g \in C\left(K_{()}, \mathbb{R}\right)$ which is $1-1$ on $K_{()} \cap V$; if $f=g \circ \pi_{0} \in C(X, \mathbb{R})$, then $\Omega_{f}=\emptyset$. To verify the details of this construction, one must compare $X$ and $C(X, \mathbb{R})$ in both models, $V$ and $V[G]$; this is described in $\S 3$ of [8].

The following theorem yields a class of examples where $E_{0}(X, \mathbb{R})$ is of first category.

5.5.2. Theorem. Let $M$ be any Banach space, and let $X=\prod_{i \in \omega} X_{i}$, where each $X_{i}$ is compact Hausdorff and has more than one point. Then $E_{0}(X, M)$ is of first category, and is dense in $C(X, M)$.

Proof. Let $P_{n}=\prod_{i=0}^{n} X_{i}$, and let $\sigma_{n}$ be the projection from $X$ onto $P_{n}$. We call a function $f$ on $X n$-supported iff $f=g \circ \sigma_{n}$ for some function $g$ on $P_{n}$. 
To prove that $E_{0}(X, M)$ is dense in $C(X, M)$, it is sufficient to show that $E_{0}(X, \mathbb{R})$ separates points. Fix two distinct points, $x, y \in X$. Since an infinite product has no isolated points, we may assume (by partitioning the index set into infinitely many infinite sets) that each $X_{i}$ has no isolated points. We may also assume that $\sigma_{0}(x) \neq \sigma_{0}(y)$. We now produce an $f$ in $E_{0}(X, \mathbb{R})$ which separates $x, y$.

Note that if $\sigma_{n}\left(\Omega_{f}\right)=P_{n}$ for all $n$, then $\Omega_{f}$ will be dense. To obtain this situation, we shall focus on the dyadic rationals. Let $D_{n}=\left\{j \cdot 2^{-n}\right.$ : $\left.0 \leq j \leq 2^{n}\right\}$; so, $D_{0}=\{0,1\}$ and $D_{1}=\{0,1 / 2,1\}$. Inductively choose $f_{n} \in C(X,[0,1])$ so that

(1) $x \in \operatorname{int}\left(f_{0}^{-1}\{0\}\right)$ and $y \in \operatorname{int}\left(f_{0}^{-1}\{1\}\right)$.

(2) $f_{n}$ is $n$-supported.

(3) $\left\|f_{n+1}-f_{n}\right\| \leq 2^{-n}$.

(4) $f_{n}^{-1}\{q\} \subseteq f_{n+1}^{-1}\{q\}$ whenever $q \in D_{n}$.

(5) $\bigcup\left\{\sigma_{n}\left(\operatorname{int}\left(f_{n+1}^{-1}\{q\}\right)\right): q \in D_{n+1}\right\}=P_{n}$.

Let $f=\lim _{n} f_{n}$. This limit exists by (3). We have $\sigma_{n}\left(\Omega_{f}\right)=P_{n}$ for all $n$ by (4)-(5), and $f$ separates $x, y$ by (1). Condition (2) allows the inductive construction of $f_{n+1}$.

Now, we prove that $E_{0}(X, M)$ is of first category in $C(X, M)$. For each $n$, let $U_{n}$ be the set of all $f \in C(X, M)$ such that for all $z \in P_{n}, f$ is not constant on $\left\{x \in X: \sigma_{n}(x)=z\right\}$. Then $U_{n}$ is dense and open in $C(X, M)$, and $\Omega_{f}=\emptyset$ whenever $f \in \bigcup_{n \in \omega} U_{n}$.

\section{References}

[1] A. V. Arkhangel'skiŭ and V. V. Fedorchuk, General Topology I, Basic Concepts and Constructions, Dimension Theory, Springer, 1990.

[2] A. Bella, A. Hager, J. Martinez, S. Woodward and H. Zhou, Specker spaces and their absolutes, $I$, preprint.

[3] A. Bella, J. Martinez and S. Woodward, Algebras and spaces of dense constancies, preprint.

[4] Y. Benyamini, M. E. Rudin and M. Wage, Continuous images of weakly compact subsets of Banach spaces, Pacific J. Math. 70 (1977), 309-324.

[5] A. Bernard, Une fonction non Lipschitzienne peut-elle opérer sur un espace de Banach de fonctions non trivial?, J. Funct. Anal. 122 (1994), 451-477.

[6] - A strong superdensity property for some subspaces of $C(X)$, prépublication de l'Institut Fourier, Laboratoire de Mathématiques, 1994.

[7] A. Bernard and S. J. Sidney, Banach like normed linear spaces, preprint, 1994.

[8] M. Džamonja and K. Kunen, Properties of the class of measure separable compact spaces, Fund. Math. 147 (1995), 261-277.

[9] P. R. Halmos, Lectures on Boolean Algebras, Van Nostrand, 1963.

[10] T. Jech, Set Theory, Academic Press, 1978.

[11] K. Kunen, Set Theory, North-Holland, 1980. 
[12] J. Martinez and S. Woodward, Specker spaces and their absolutes, II, Algebra Universalis, to appear.

[13] J. van Mill, A homogeneous Eberlein compact space which is not metrizable, Pacific J. Math. 101 (1982), 141-146.

[14] M. E. Rudin and W. Rudin, Continuous functions that are locally constant on dense sets, J. Funct. Anal. 133 (1995), 120-137.

[15] S. J. Sidney, Some very dense subspaces of $C(X)$, preprint, 1994.

[16] R. Sikorski, Boolean Algebras, Springer, 1964.

Department of Mathematics

University of Wisconsin

Madison, Wisconsin 53706

U.S.A.

E-mail: jhart@math.wisc.edu

kunen@cs.wisc.edu

Received 22 May 1995;

in revised form 14 November 1995 